\title{
A systematic global stocktake of evidence on human adaptation to climate change
}

\begin{abstract}
Assessing global progress on human adaptation to climate change is an urgent priority. Although the literature on adaptation to climate change is rapidly expanding, little is known about the actual extent of implementation. We systematically screened $>48,000$ articles using machine learning methods and a global network of 126 researchers. Our synthesis of the resulting 1,682 articles presents a systematic and comprehensive global stocktake of implemented human adaptation to climate change. Documented adaptations were largely fragmented, local and incremental, with limited evidence of transformational adaptation and negligible evidence of risk reduction outcomes. We identify eight priorities for global adaptation research: assess the effectiveness of adaptation responses, enhance the understanding of limits to adaptation, enable individuals and civil society to adapt, include missing places, scholars and scholarship, understand private sector responses, improve methods for synthesizing different forms of evidence, assess the adaptation at different temperature thresholds, and improve the inclusion of timescale and the dynamics of responses.
\end{abstract}

T he Paris Agreement commits parties to track climate adaptation progress ${ }^{1,2}$. In response, there have been consistent and increasingly urgent calls for robust, systematic and transparent assessments of adaptation progress, which include the regular stocktake of insights from empirical research ${ }^{1,3}$. Understanding if and how adaptation is taking place is critical for decision-making. Assessments of adaptation progress can facilitate the sharing of best practices, identify gaps, support the prioritization of adaptation finance and map evidence across regions and sectors ${ }^{3-5}$.

In the absence of systematic, global data on adaptation practices, adaptation actions documented in the academic literature provide a valuable complement to efforts to track adaptation on the ground (see Supplementary File 1 for a background on adaptation tracking and global adaptation mapping). Other studies assessed adaptation planning and policy at the regional ${ }^{6-14}$, national ${ }^{15-18}$ and subnational ${ }^{19-23}$ levels, using information from national communications ${ }^{24-26}$, local climate change action plans ${ }^{22,23,27,28}$, adaptation project proposals ${ }^{29}$ and peer-reviewed literature ${ }^{20}$. Systematic approaches to synthesizing these and other types of adaptation evidence are emerging and are crucial for learning about what adaptation measures work, under what conditions, for whom and why ${ }^{1,30-34}$. However, to date, few syntheses of adaptation actions are documented in the academic literature ${ }^{30-32}$. The literature on climate change adaptation is vast and fast-growing, and spread across disparate academic communities ${ }^{32,35-37}$. Relatively few of these papers document adaptation actions that have actually taken place, but separating out the studies that report on adaptation actions (rather than, for example, vulnerability assessments or studies that model the potential for actions to address climate change or document the barriers that prevent adaptation) is a monumental task. Moreover, it is impossible to document and capture all-or even a fraction of-adaptation-related activities occurring on the ground, and there are therefore no reliable estimates of what proportion of adaptation activities are documented or reflected in the academic literature (Supplementary File 1). As a result, this knowledge base has remained under-utilized, despite the opportunities it presents to better understand the adaptation activities to date and to inform future responses and research.

This article presents a comprehensive, systematic and global review of the academic literature that documents implemented human adaptation actions in response to climate change. We focus on empirical studies that report observed adaptation-related responses (hereafter referred to as 'responses'), which reflects our aim to capture adaptations with the potential to directly reduce climate risk, and acknowledges that responses do not necessarily lead to reduced risk. In doing so, we focus on a specific subset of adaptation literature that reflects observed and implemented responses rather than processes of decision-making, adaptation governance and planning.

As the volume of literature makes reliable synthesis via conventional assessment methods impossible, we draw on two recent approaches in information science: machine learning ${ }^{38-40}$ and collaborative networks ${ }^{41-44}$. Machine learning techniques allow us to rapidly sort thousands of documents and capture the breadth of adaptation literature to an extent that would not be feasible using manual methods ${ }^{32,36,37,39,40,45}$. We used supervised machine learning to screen 48,816 articles published between 2013 and 2019 and identified 1,682 articles that met our inclusion criteria (Methods and Extended Data Figs. 1 and 2). We developed a network of 126 global experts in adaptation research to collaboratively and systematically extract information and evidence from these articles, asking: What climate hazards are driving responses? Who is responding? What types of responses are documented? Is adaptation reducing climate change risk? Are adaptations transformational?

\section{Stocktaking global adaptation responses}

Academic studies report adaptation responses across all global regions, with the greatest number of papers reporting responses in Asia (35\% of articles) and Africa (32\%) (Fig. 1 and Table 1). A minority of publications focused on Central and South America (6\%) or Small Island States (2\%). Reporting in Africa and Asia is dominated by literature from southern and eastern Africa and South Asia, with limited documentation from Central, Western and Northern Africa and from Northern, Central and Western Asia.

Responses were most frequently documented in the context of food and agriculture (close to $66 \%$ of all the articles), which was consistent across all regions except for Oceania and Europe, for which health (both) and adaptation in urban areas (Europe) were more prominent (Fig. 1). We found geographical gaps in evidence (that is, far fewer papers) from South America, Central and North Africa, 


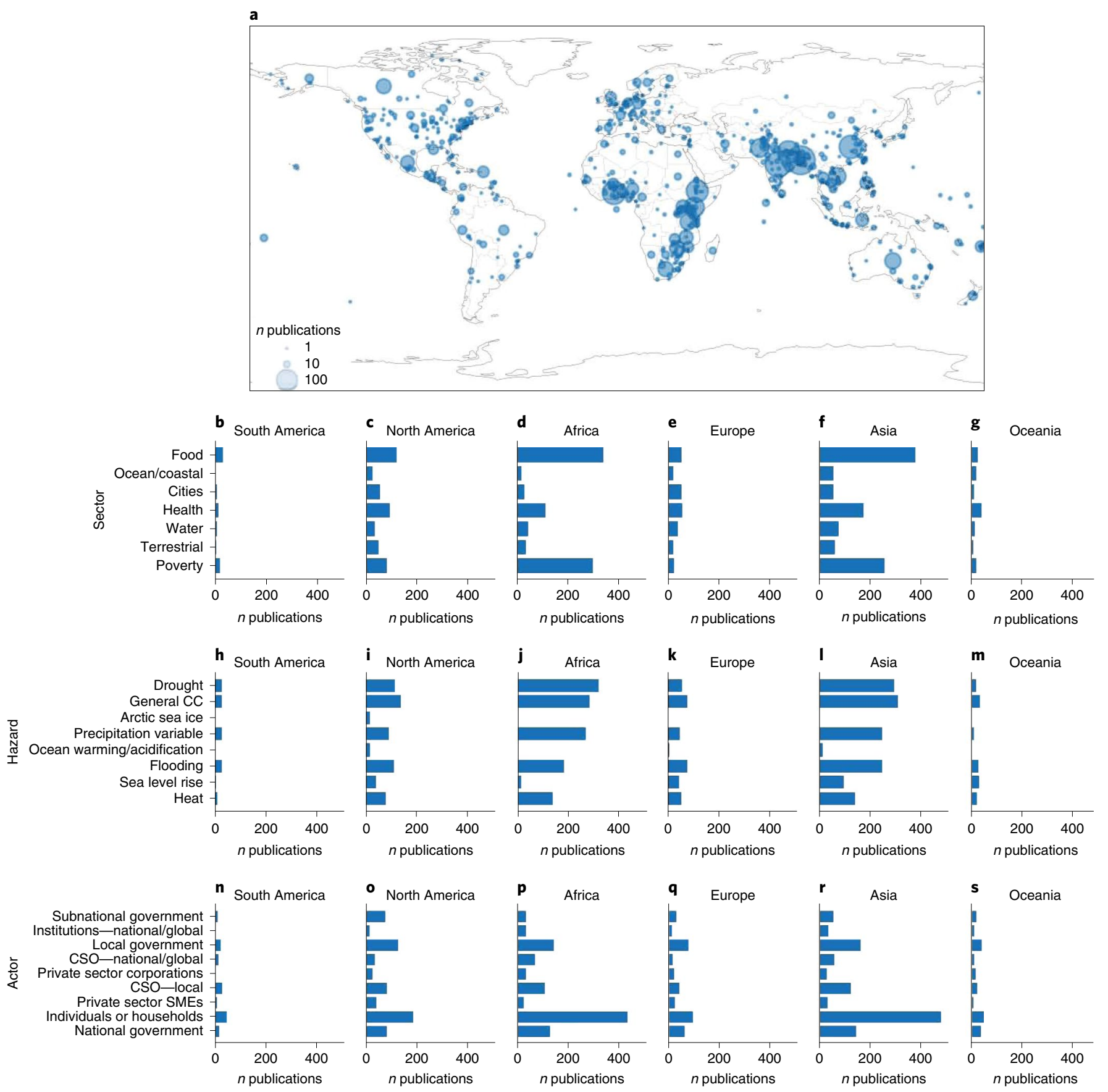

Fig. 1 | The geographical and sectoral distribution of the 1,682 articles included in the analysis. a-s, Geographical distribution of included studies (a) and descriptive summary of articles included in this review (b-s). Bar graphs show the total number of publications by global region for categories of sector $(\mathbf{b}-\mathbf{g})$, hazards $(\mathbf{h}-\mathbf{m})$ and actors $(\mathbf{n}-\mathbf{s})$. Bubbles in a reflect the number of publications based on the location mentioned in the study; bubbles shown in the centre of countries reflect articles with a national focus or are unspecified beyond the national level. SME, small and medium enterprise; CC, climate change; CSO, civil society organization.

the Middle East and Central Asia (Fig. 1). Health risks of climate change were among the top three issues that motivated responses across all the regions. Poverty and livelihood-related responses were particularly common in Africa, Asia and North America. In North America and Europe, there was a relatively strong reporting of urban responses (Fig. 1). Percentages reported throughout this section do not sum to $100 \%$, unless otherwise noted, as articles could describe actions taken in multiple regions or sectors, by multiple actors and in response to multiple hazards.

\section{Climate hazards driving adaptation responses}

Many responses were motivated by the observed or predicted general impacts of climate change ( $58 \%$ of the articles; Table 1 ). Of those that noted particular hazards as motivators, drought (54\%), extreme precipitation and inland flooding (43\%), and precipitation variability (44\%) were most common (Fig. $1 \mathrm{~h}-\mathrm{m})$. Drought and precipitation variability are particularly important motivators of responses in Africa and Central and South America, for example, through the uptake of new forms of agriculture ${ }^{42,46,47}$, food systems ${ }^{48-50}$ and the 


Table 1 | Distribution of article by categories of hazard, actor,
sector and type of response
$\begin{array}{lll}\text { Indicator Category } & \begin{array}{l}\text { Number of } \\ \text { articles }^{\mathrm{a}}\end{array} & \begin{array}{l}\text { Share of } \\ \text { database } \\ (\%)^{b}\end{array}\end{array}$

Hazards

\begin{tabular}{|c|c|c|}
\hline Sea level rise & 253 & 15 \\
\hline $\begin{array}{l}\text { Extreme precipitation and inland } \\
\text { flooding }\end{array}$ & 726 & 43 \\
\hline $\begin{array}{l}\text { Increased frequency and intensity of } \\
\text { extreme heat }\end{array}$ & 475 & 28 \\
\hline Precipitation variability & 744 & 44 \\
\hline Drought & 897 & 53 \\
\hline $\begin{array}{l}\text { Rising ocean temperature and ocean } \\
\text { acidification }\end{array}$ & 51 & 3 \\
\hline Loss of Arctic sea ice & 28 & 2 \\
\hline General climate impacts & 973 & 58 \\
\hline Other & 495 & 29 \\
\hline
\end{tabular}

Actors

International or multinational

governance institutions

Government (national)

129

Government (subnational)

Government (local)

Private sector (corporations)

Private sector (SME)

Civil society (international,

multinational, national)

Civil society (subnational or local)

Individuals or households

Other

$\begin{array}{lll}\text { Individuals or households } & 435 & 26 \\ \text { Other } & 1,374 & 82 \\ & 226 & 13\end{array}$

Sectors

Terrestrial and freshwater

ecosystems

Ocean and coastal ecosystems

Water and sanitation

Food, fibre and other ecosystem

products

Cities, settlements and key

infrastructure

Health, well-being and communities

Poverty, livelihoods and sustainable development

Type of response

$\begin{array}{lll}\text { Behavioural and/or cultural } & 1,259 & 75 \\ \text { Ecosystem-based } & 840 & 50 \\ \text { Institutional } & 707 & 42 \\ \text { Technological and/or infrastructure } & 1,048 & 62\end{array}$

${ }^{\mathrm{a}}$ Categories are not mutually exclusive and sum to more than $1,682 .{ }^{\mathrm{b}} \mathrm{Categories}$ are not mutually exclusive and sum to more than $100 \%$.

household-level water supply in cities ${ }^{51,52}$. In Bolivia, Guatemala and Kenya, for example, the threat of droughts and precipitation variability have spurred changes in food systems ${ }^{53,54}$. Flooding and rising sea levels most commonly drive responses in Small Island States, compelling people to prepare inland and coastal flood management infrastructure, implement new building codes and develop hazard maps and early warning systems ${ }^{55-57}$. In cities worldwide, flooding and sea level rise are most frequently cited as key motivating hazards $s^{17,58-60}$. For example, increasing flood risks are prompting European countries with large urban areas to diversify, coordinate and align flood risk management strategies ${ }^{17}$. Although not commonly identified as a major driver of responses, extreme heat $(28 \%$ of articles) appears to play a role in motivating responses across most regions and sectors ${ }^{19,61-63}$.

\section{Level and actors responding to climate hazards}

Responses occur at multiple levels of social organization, from individual farmers and urban households, to water, electric and transportation utilities and managers, and to international institutions ${ }^{24,55,64-69}$. However, the vast majority of responses documented in the academic literature are undertaken at the local level, and by households or individuals in particular ( $82 \%$ of all the articles) (Fig. 1 n-s, Table 1 and see Supplementary File 1 for a reflection on how the results in the academic literature may differ from those of other data sources). Household or individual-level responses are frequently reported in the context of food, health and poverty in Africa and Asia ${ }^{53,70,71}$. For example, studies in Ghana and Uganda observe farming households that respond to drought by diversifying and irrigating crops, planting drought-tolerant crops and livelihood diversification strategies, which include migration ${ }^{70,71}$, whereas in Kenya, households are diversifying livelihoods through farming and ecotourism ${ }^{53}$.

Local governments are also prominent actors (Table 1), particularly in large urban areas. In Ibadan (Nigeria), state governments established urban agriculture programmes ${ }^{72}$, and city governments in Quito (Ecuador) and Lima (Peru) constructed large water reservoirs and water-treatment plants to mitigate water shortages for urban populations ${ }^{73}$. Responses at the level of national governments also receive substantial attention ${ }^{74}$. Caribbean governments, for example, have instituted education and capacity building programmes $^{12}$. In Central and South America and Small Island States, a relatively large percentage of papers describe actions by local civil society, as in Bolivia, where local community organizations support practices such as composting and climate smart agriculture ${ }^{53}$. Reporting in the academic literature on private sector engagement in responses is low across all regions ${ }^{69,75}$ except for Australasia and Europe, where, for instance, tourism companies initiated safeguards to protect the industry against glacier thinning and decline in snowfall ${ }^{73}$.

\section{Types of responses documented}

The vast majority of responses documented in the academic literature globally are behavioural in nature $(75 \%)$, with many also technical and/or infrastructural (63\%) and institutional (42\%) (Fig. 2 and Table 1). Behavioural responses include actions such as: people making changes to their homes and land to protect them from floods, fires and heat ${ }^{68}$, relocating or migrating from hazards ${ }^{76,77}$ or adopting crops and livestock that are adapted to drought, pests and encroaching salinity ${ }^{78-82}$. Individuals shift to other economic and livelihood activities, abandoning fishing for farming ${ }^{83}$, or change food consumption practices to cope with environmental risks. In Africa and Asia, farmers commonly use drought-tolerant plant and animal species, water and soil management practices, and diversified income streams to spread risks and adjust to shifting climate conditions $^{80,84-89}$. Technical and infrastructural responses are also common, most notably in Europe and in cities, particularly in the water sector ${ }^{90,91}$. Institutional responses, such as creating policies, programmes, regulations and procedures and establishing formal and informal organizations-for example, social support groups, climate insurance services ${ }^{92}$, capacity building and financial 


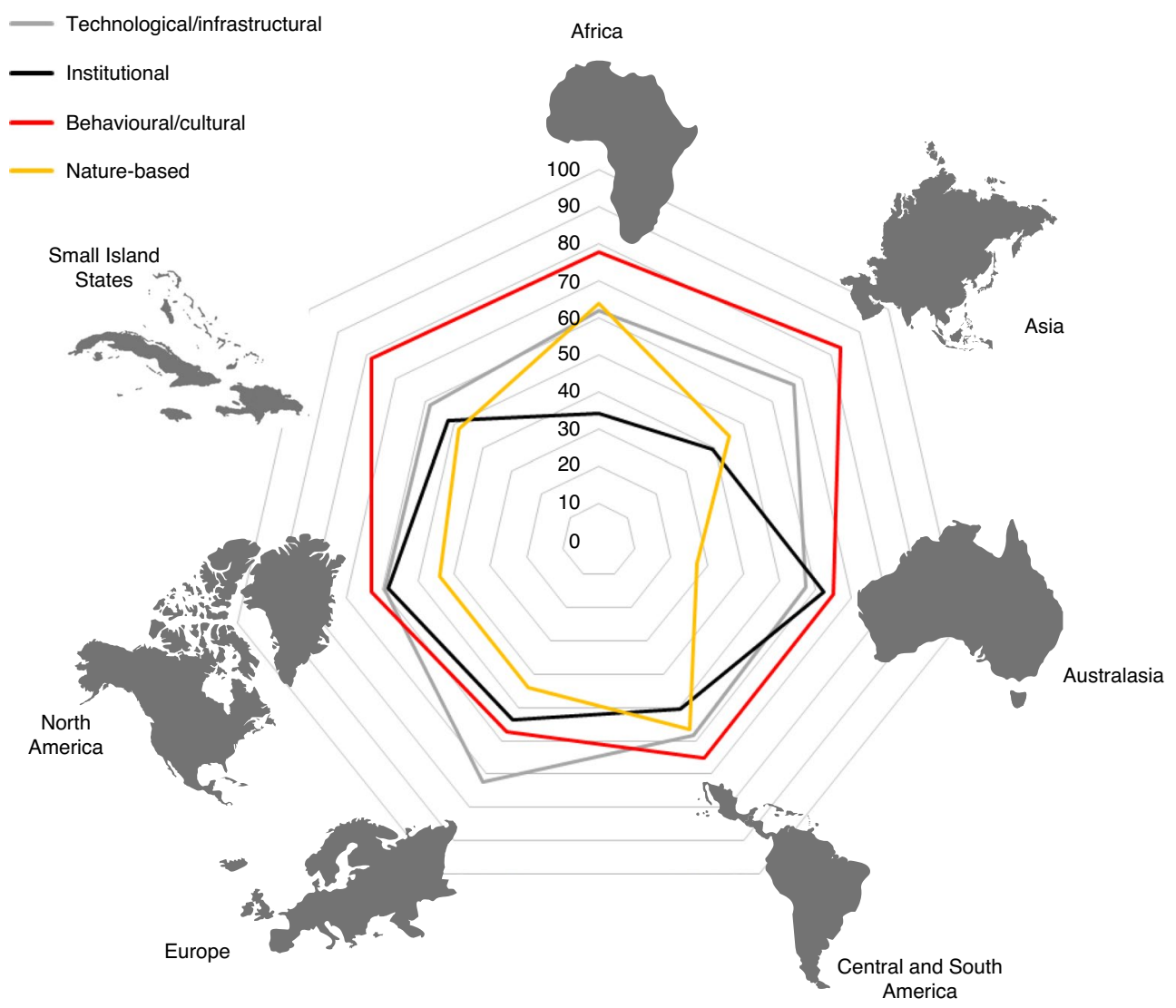

Fig. 2 | Types of adaptation responses, by global region. Radar axes reflect the percentage of articles that mention each type of adaptation response over the total number of articles for that region. Adaptation types are not exclusive; articles frequently reported responses that involved multiple types of adaptation, for example, the installation of urban green roofs for cooling (nature-based and technological) or government-supported planting of drought-resistant seeds among subsistence farmers (behavioural and institutional).

assistance programmes-are reported most frequently in the food and health sectors and in cities. Institutional adaptations often support other responses, such as extension services designed to enable farmer uptake of drought-tolerant crops $^{93}$ or public education for flood risk preparedness ${ }^{94}$. Ecosystem- or nature-based responses (50\% of all articles), such as the natural regeneration of plant species $^{78}$, intercropping and mulching are used across all regions, most notably in Africa and Central and South America ${ }^{95-97}$.

\section{Evidence of climate risk reduction due to adaptation}

Given that adaptation aims to limit climate risks by reducing vulnerability and exposure to climate hazards, understanding the extent to which responses have contributed to risk reduction is critical to evaluate adaptation effectiveness and inform future action. Yet the vast majority of the papers we reviewed lacked detailed accounting of how and to what extent responses lower climate risk, as the authors often assumed or implied risk reduction.

The results from the coding indicate that $62 \%$ of papers $(n=1,044)$ provided implicit or explicit evidence that adaptation activities were reducing the risk or vulnerability (Question 5.1, Supplementary File 6), but only 58 papers $(3.4 \%)$ indicated that the risk reduction outcomes of adaptation responses were formally assessed after implementation (Question 4.1). We conducted a further analysis of this subset of papers that were reported to include a formal assessment of risk reduction to examine the current state of empirical evidence on risk reduction (see Supplementary File 2 for further details and methods). Among this subset, 30 papers $(1.8 \%$ of all academic studies in our database) present primary evidence of risk reduction, for example, improved food security and health outcomes measured through indicators such as increased agricultural yields and caloric intake ${ }^{98}$. These studies applied either quantitative (15 articles) or qualitative (11) methods to assess risk reduction, and a minority (4) used mixed methods. A further nine articles quantitatively assessed the improvements in adaptive capacity, but with no clear evidence of changes in the risk outcomes. The remaining 19 (of 58) papers assumed risk reduction outcomes based on secondary evidence or theories of change. Among the possible explanations for the limited evaluative evidence in the literature are technical challenges related to risk reduction evaluation, including the lead time until responses show outcomes in terms of risk reduction, the difficulty of attributing such outcomes to the studied responses and the difficulty of measuring avoided impacts or risks ${ }^{3,99,100}$. Additionally, limited evaluative evidence could be related to differences among coders in the understandings of risk reduction, assumptions regarding outcomes based on related but non-climate change-specific literature and the difficulty of conceptualizing risk and factors that lead to risk reduction.

Notably, some adaptation responses may be counterproductive, with mixed outcomes for risk reduction, especially over the longer term ${ }^{101}$. This is mentioned in approximately $33 \%$ of papers in our sample (Question 5.3, Supplementary File 6). For example, there is some evidence that watershed management responses, such as water harvesting, may reduce the water supply risk in the watershed where water harvesting happens, but may have negative outcomes downstream for particular user groups or at longer timescales ${ }^{102,103}$. Migration provides another example of a response to climatic and 
Table 2 | Scenarios of low, medium and high transformational adaptation across four dimensions

Evidence of transformational adaptation

\begin{tabular}{|c|c|c|c|}
\hline $\begin{array}{l}\text { Dimensions of } \\
\text { transformational } \\
\text { adaptation }\end{array}$ & Low & Medium & High \\
\hline Overall & $\begin{array}{l}\text { Adaptation is largely sporadic and consists } \\
\text { of small adjustments to business as usual. } \\
\text { Coordination and mainstreaming are limited } \\
\text { and fragmented. }\end{array}$ & $\begin{array}{l}\text { Adaptation is expanding and } \\
\text { increasingly coordinated, and } \\
\text { includes wider implementation and } \\
\text { multilevel coordination. }\end{array}$ & $\begin{array}{l}\text { Adaptation is widespread and } \\
\text { implemented at or very near its full } \\
\text { potential across multiple dimensions. }\end{array}$ \\
\hline Depth & $\begin{array}{l}\text { Adaptations are largely expansions of } \\
\text { existing practices, with minimal change in } \\
\text { underlying values, assumptions or norms. }\end{array}$ & $\begin{array}{l}\text { Adaptations reflect a shift away } \\
\text { from existing practices, norms or } \\
\text { structures to some extent. }\end{array}$ & $\begin{array}{l}\text { Adaptations reflect entirely new practices } \\
\text { that involve deep structural reform, } \\
\text { complete change in mindset, major shifts } \\
\text { in perceptions or values and changing } \\
\text { institutional or behavioural norms. }\end{array}$ \\
\hline Scope & $\begin{array}{l}\text { Adaptations are largely localized and } \\
\text { fragmented, with limited evidence of } \\
\text { coordination or mainstreaming across sectors, } \\
\text { jurisdictions or levels of governance. }\end{array}$ & $\begin{array}{l}\text { Adaptations affect wider } \\
\text { geographical areas, multiple areas } \\
\text { and sectors or are mainstreamed } \\
\text { and coordinated across multiple } \\
\text { dimensions. }\end{array}$ & $\begin{array}{l}\text { Adaptations are widespread and } \\
\text { substantial, and include most possible } \\
\text { sectors, levels of governance and actors. }\end{array}$ \\
\hline Speed & Adaptations are implemented slowly. & $\begin{array}{l}\text { Adaptations are implemented } \\
\text { quickly. }\end{array}$ & $\begin{array}{l}\text { Change is considered rapid in a given } \\
\text { context. }\end{array}$ \\
\hline Limits & $\begin{array}{l}\text { Adaptations may approach but do not exceed } \\
\text { or substantively challenge soft limits. }\end{array}$ & $\begin{array}{l}\text { Adaptations may overcome some } \\
\text { soft limits, but do not challenge or } \\
\text { approach hard limits. }\end{array}$ & $\begin{array}{l}\text { Adaptations exceed many soft limits and } \\
\text { approach or challenge hard limits. }\end{array}$ \\
\hline
\end{tabular}

Transformational adaptation does not imply the adequacy or effectiveness of responses (a low level of transformational adaptation may be sufficient for some climate risks, and a high level of transformational adaptation may be insufficient to offset other climate risks). Dimensions (depth, scope, speed) are adapted from Termeer et al. ${ }^{108}$.

non-climatic hazards, for which there is mixed evidence of risk reduction, especially in Asia (37 studies), Africa (21), South and Central America (7), and North America (6). In some cases, migration may have negative repercussions. For example, labour may be reduced in communities from which individuals migrate, with the result that female heads of households experience increased demands with less ability to share labour ${ }^{104-107}$.

\section{Evidence of transformational adaptation}

As the impacts of climate change become more severe, adaptation may need to be more transformational than incremental, with responses going beyond business as usual or incremental changes to activities that change the fundamental attributes of socio-ecological systems ${ }^{108-114}$. To assess the evidence of transformational adaptation in the documented responses, we drew on a typology developed by Termeer et al., which outlines three dimensions of transformative governance: depth, scope and speed ${ }^{108}$. Depth describes the novelty of an action, scope the geographical or sectoral breadth and speed the time taken to implement. We added a fourth component that asked to what extent adaptation actions approach or overcome the limits known to constrain adaptation. We operationalized this typology to assess evidence within our database of transformational adaptation for global regions and sectors (Table 2; see also Supplementary File 3 for detailed methods, categories, and definitions). We categorized evidence of transformational adaptation for the four dimensions within our typology (depth, scope, speed, limits) as high, medium, or low (Table 1 in Supplementary File 3). Evidence of a high transformational adaptation involves an overall regional or sectoral profile of novel adaptations at large scales or across numerous sectors, implemented quickly, that overcome or reduce constraints on adaptation. Conversely, evidence of low transformational adaptation describes an overall profile of adaptation that is largely localized, implemented slowly, involves small adjustments to business as usual and is constrained by barriers to adaptation.
Across all regions and sectors, the depth of responses is low, with few exceptions, as they involve minor adjustments to business as usual rather than transformation, and short-term responses to extreme weather events more than long-term proactive change. Alterations in farming practices (for example, irrigation, crop variety and timing) or infrastructural modifications (for example, building elevation) fall into this category. Less commonly reported are high-depth responses, such as the permanent relocation of a village or a large-scale, multistakeholder effort to create a resource governance system ${ }^{115-117}$. Documented responses also tend to be small in scope, and focused on a single sector or a small geographi$\mathrm{cal}$ area. Autonomous responses by individuals to deal with heat, for example, tend to be of small scope $e^{61,118}$. Conversely, a national plan to address numerous aspects of climate change is of large scope ${ }^{119}$. Individual actions can be large scope when adopted by numerous individuals or households across a relatively large geographical region or when actions affect numerous aspects of life rather than focusing on a single hazard. The speed of adaptation is often not documented explicitly, but ranges from fast responses that occur in less than a year (for example, using shade or fans in a heat wave or changing the timing of crop planting) to slow responses that require more than a decade of planning and execution. Some fast actions may occur quickly at an individual level, but still be slow to spread to other individuals (for example, the uptake of a new irrigation technique by farmers). Numerous constraints that limit the ability of various actors to respond were noted ( $80 \%$ of studies describe constraints), and there is little evidence of these constraints being overcome.

The overall transformative potential of adaptations documented in the academic literature across most global regions and sectors is low (Fig. 3). Some adaptations exhibit a high depth, scope and speed, and challenge limits ${ }^{120}$, but these are uncommon. In fact, the results suggest there may be trade-offs between the scope of responses on the one hand and the speed of implementation on the other ${ }^{107}$, perhaps due to the long timelines involved in coordinating 


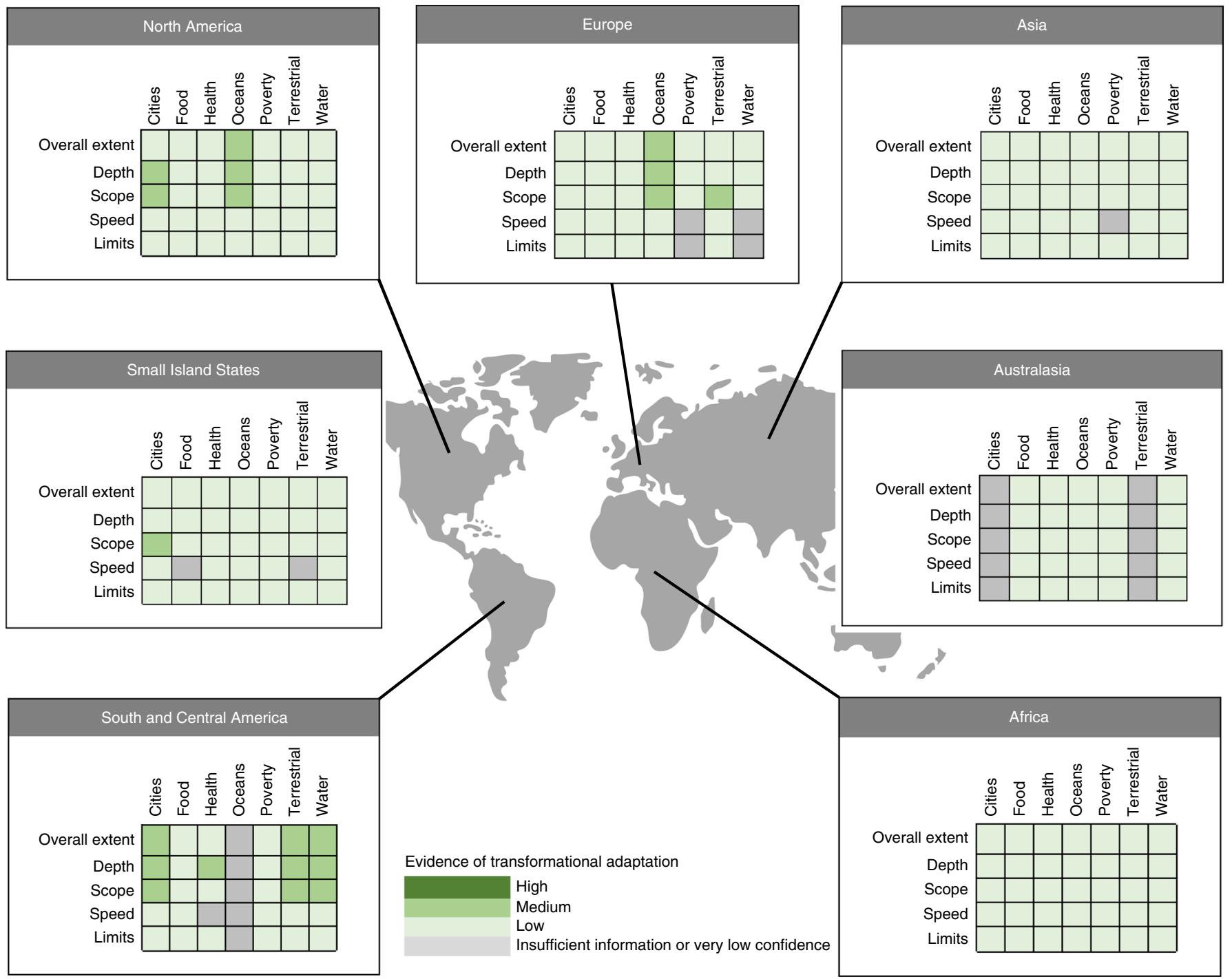

Fig. 3 | Evidence of transformational adaptation by sector and region. The overall profile across global sectors and regions indicates that evidence for transformational adaptation is low. We found no sector or region with evidence of a high overall transformational adaptation, and few with evidence of medium levels of transformational adaptation. Evidence across some sectors and regions was insufficient for assessment. Transformational adaptation does not imply adequacy of adaptations to reduce risk, which is currently not methodologically feasible or available in the literature. Transformational adaptation here is based on the assessment of the scope, speed, depth and challenges to adaptation limits of responses reported in the academic literature. The methodology is provided in Supplementary File 2.

or executing large-scale measures. Further research is needed to explore the implication that soft limits impeded the ability to implement widespread change with the urgency required for adaptation.

\section{Discussion}

Ultimately, adaptation intends to reduce the adverse effects of climate change and, in some cases, to take advantage of new opportunities. Although our results find widespread documentation of adaptation-related responses in the academic literature, there is little evidence as to whether the responses are reducing climate risk. We identified only 1,682 articles that met our inclusion criteria from $>48,000$, which highlights that only a small fraction of the broader adaptation literature $(<5 \%)$ reports on implemented adaptation responses. There are also concerning gaps that arise from our results, such as a relative scarcity of reported transformative adaptations in cases for which the current and projected risks are high, and a lack of evidence that well-documented limits to adaptation are being challenged or overcome. These knowledge gaps reflect the substantial and recognized difficulties involved in measuring the actual (when responding to observed risks) and potential (when responding to projected risks) effectiveness of a wide range of adaptation responses.

The absence of evidence of risk reduction in the academic literature that documents implemented adaptation actions does not necessarily imply that no risk reduction is taking place. Adaptation actions are documented beyond the academic literature as well (for example, in the grey literature). It is possible that there is more evidence of risk reduction in these other literatures, so to evaluate that literature is an important next step for global adaptation stocktaking. We conducted an internal expert elicitation exercise to assess confidence in the extent to which our results reflect real-world trends in the evidence for transformational adaptation, which highlights reporting bias but broadly supports a pattern of low overall evidence on transformational adaptation (see Supplementary Files 3 Table 3 and 4). The absence of empirical evidence on risk reduction that we identified in our database was not just a matter of delay between 


\section{Box 1 | Moving forward: eight key priorities for global adaptation research}

\begin{abstract}
Assess effectiveness of adaptation response. Few studies attempt to assess outcome measures ${ }^{128}$. Ultimately, and in most cases, adaptation success results in avoided harm at some point in the future. This is intrinsically difficult to measure, but it is possible to assess change in climatological, ecological and human health outcome variables, such as flood damage, crop yield and health impacts. Introducing effective monitoring of these variables at the start of adaptation programmes, ideally in a comparative way with counterfactuals, would allow assessment of the response effectiveness, and at least observed, current benefits. Dedicated funding, training, monitoring and research streams are needed to overcome barriers to the development and implementation of frameworks for effectiveness assessments. There is considerable potential to draw on existing tools, such as theories of change, and from synthesizing insights from evaluations in the non-academic literature to increase the consideration of how responses are affecting transitions towards risk reduction and minimizing the risk of maladaptation.
\end{abstract}

Enhance understanding of limits to adaptation and adaptation adequacy. Investigation of adaptation limits remains underdeveloped within adaptation research, yet it is important to track if and how implemented adaptation addresses or approaches limits, that is, whether it is adequate in the face of climate change. Frameworks to assess adaptation adequacy remain elusive, which is unlikely to change within the timescales available for rapid climate action. It is clear, however, that the transformational nature of adaptation globally is low, although this is highly variable, with a substantial potential to extend the depth, scope and speed of adaptations, and to begin to overcome barriers and approach limits. A precautionary approach and (limited) available evidence suggests that we assume our current response is inadequate. More research is needed to understand why complacency persists and why we are not learning how to overcome well-known barriers to adaptation ${ }^{107}$. An assessment of transformational adaptation is an imperfect but useful tool to gauge progress on adaptation across scales.

Enable individuals and civil society. Much global adaptation discourse focuses on institutional adaptation and addressing governance barriers with a focus on formal institutions and state actors. Although critical, this narrative can divert recognition and resources from the importance of autonomous adaptation by individuals and households, particularly in the Global South. To enable long-term adaptation, it is critical to recognize and test different incentive mechanisms that enable behavioural change towards adaptation, which include insights from environmental psychology ${ }^{129}$. It is also important to assess collective action for adaptation (that is, autonomously organized adaptation action among social groups) as this holds the potential to facilitate transformational adaptation through social change $\mathrm{e}^{110}$.

Include missing places, scholars and scholarship. Adaptation research is notably greater in Africa and Asia, consistent with global

the implementation and realization of risk reduction (although that is certainly relevant), but also a matter of the lack of engagement with pathways of risk reduction more broadly. Another limitation is that we do not map responses against projected risk from climate hazards. To assess the extent to which responses are addressing key climate hazards is critical to identify areas of progress and gaps in risk reduction. Finally, our analysis suggests that synthesizing different sources of information is needed at the regional and sectoral levels, given the observed high degree of interregional variation. areas of greatest climate vulnerability. However, important gaps remain, which include Central and South America, Central Asia, Central and North Africa, and the Middle East, where vulnerability is high but adaptation research is comparatively sparse.

Understand private sector responses. There is relatively little academic literature that assesses responses within the private sector $^{75}$. Although this may be proprietary (not reported) or published elsewhere, the integration of private sector experiences and insights with institutional and public responses will be critical to a comprehensive assessment of adaptation.

Improve methods to synthesize different forms of evidence. The work presented here-despite requiring a huge international collaborative effort-captures only those adaptations reported in the academic literature. Although we originally aimed (and attempted) to integrate grey literature, it was not technically or logistically feasible. Systematically synthesizing insights from grey literature, adaptation practice and Indigenous and local knowledge remains a grand challenge for adaptation evidence synthesis. This is compounded by a rapidly expanding and diverse literature base on adaptation. There is a critical need for innovation of the conceptual and methodological tools to keep up with and synthesize the diverse knowledge on adaptation.

Assess adaptation at different temperature thresholds. Our findings are consistent with evidence that the vulnerability assessment literature remains largely temperature agnostic ${ }^{130}$; the literature on adaptation implementation is likewise underdeveloped with regards to outcomes under different temperature scenarios, and disconnected from mitigation and warming estimates. This disconnect is partly because mitigation and warming estimates do not translate hazard trends into environmental and societal impacts that are a prerequisite to understanding and discussing adaptation needs and responses. As a start, adaptation studies could consider how limits to adaptation or the effectiveness of a given range of measures will differ by degree and speed of warming.

Improve inclusion of timescale and dynamics of responses. Greater understanding is needed on the durability of documented adaptation responses, how long it takes for their benefits to accrue and to whom they accrue. Such longitudinal understanding would help inform policies at various scales about the responses available now, those that need to be planned and the synergies or trade-offs between various types of responses over time. For instance, are the documented benefits maintained over time as hazards, vulnerability and exposure continue to change or do different groups begin to 'win' and 'lose'? To what extent are benefits and risks flexible to changing hazards and societal shifts? Future adaptation research and assessments should consider explicitly integrating these questions, and in particular consider longitudinal analyses to assess how adaptation evolves over time.

Nevertheless, our results highlight the stark inadequacy of the current methods and evidence base available to assess the effectiveness of responses in terms of risk reduction ${ }^{120}$. Here we argue that the inability to confidently and systematically gauge the effectiveness of adaptation-related interventions critically limits the ability to report on and galvanize adaptation globally. To critically assess the effectiveness of adaptation actions and their potential or actual risk reduction may require different approaches to adaptation research, which include longitudinal studies to assess performance over 
time, more interdisciplinary and transdisciplinary collaboration to assess multiple facets of performance and a greater incorporation of Indigenous knowledge and local knowledge to assess the effects of adaptation on communities and local ecosystems.

We identified additional evidence gaps that pertain to adaptation-related responses. Geographically, the evidence primarily documents responses in North America, Europe, parts of Africa (largely anglophone) and Asia (largely southeast). Gaps in the evidence are particularly notable in vulnerable regions in South America, Central and North Africa, and Central Asia. There remains relatively little documentation in the peer-reviewed literature on responses within the private sector. More broadly, terminology within the adaptation literature we reviewed is largely disconnected from frequently used terms in the impacts literature; for example, discussion of barriers rather than of adaptation limits, and a negligible focus on the implications of different warming levels on adaptation needs or sufficiency. The persistent lack of integration of concepts, terminology and methods between climate impacts, vulnerability, adaptation and mitigation research constrains progress on assessing how adaptation responses will interact with mitigation responses to reduce climate risk.

Recent review papers document the rapid rise in scholarship on climate change adaptation in recent years ${ }^{32,121,122}$. Our paper complements this literature by focusing specifically on the documentation of implemented adaptations in the academic literature and beyond responses by institutions. A review of climate change vulnerability research, for example, found that only about $30 \%$ of papers covered multiple systems and/or sectors ${ }^{123}$, which is similar to our finding that the research on adaptation responses tends to have a single-sector focus. Several review papers document the geographical distribution of authors in the field of climate change and adaptation, with concentrations in the United States, Canada, Europe, the United Kingdom and Australia ${ }^{121,122}$. This authorship distribution is not reflective of our mapping of the study areas of literature that documents adaptation responses, which is largest in Asia and Africa. A recent bibliometric review of adaptation literature revealed a growing number of studies on food security and agriculture in the $2016-2020$ period $^{122}$, which is reflected in the large number of papers on food-related adaptation responses documented here.

The collaborative network approach used in this study represents a way forward for large-scale synthesis efforts to overcome barriers of scale. Including a diverse set of collaborators, both junior and senior researchers, also ensures diversity in expertise, viewpoints and geography to help ground results. There is potential in the future to blend this approach with additional machine learning techniques to enable even larger comparisons or more fine-grained data extraction. These methods complement the emerging citizen science approaches, which show potential for documentation of adaptation responses not readily captured in the published literature ${ }^{124-127}$. Our study highlights that new approaches to evidence synthesis are increasingly necessary to take stock of the current conditions and to inform interdisciplinary climate solutions.

We identify eight key priorities for global adaptation research moving forward (Box 1). These recommendations are drawn from key insights that emerged from our results, combined with our collective reflection on critical gaps in research and knowledge that constrain the assessment and learning on progress towards adapting to climate risks globally.

\section{Online content}

Any methods, additional references, Nature Research reporting summaries, source data, extended data, supplementary information, acknowledgements, peer review information; details of author contributions and competing interests; and statements of data and code availability are available at https://doi.org/10.1038/ s41558-021-01170-y.

Received: 30 October 2020; Accepted: 31 August 2021;

Published online: 28 October 2021

\section{References}

1. Berrang-Ford, L. et al. Tracking global climate change adaptation among governments. Nat. Clim. Change 9, 440-449 (2019).

2. Tompkins, E. L., Vincent, K., Nicholls, R. J. \& Suckall, N. Documenting the state of adaptation for the global stocktake of the Paris Agreement. WIREs Clim. Change 9, e545 (2018).

3. Ford, J. D. et al. Adaptation tracking for a post-2015 climate agreement Nat. Clim. Change 5, 967-969 (2015).

4. Magnan, A. K. Metrics needed to track adaptation. Nature 530, 160 (2016).

5. Ebi, K. L., Boyer, C., Bowen, K. J., Frumkin, H. \& Hess, J. Monitoring and evaluation indicators for climate change-related health impacts, risks, adaptation, and resilience. Int. J. Environ. Res. Public Health 15, 1943 (2018).

6. Biesbroek, R. \& Delaney, A. Mapping the evidence of climate change adaptation policy instruments in Europe. Environ. Res. Lett. 15, 083005 (2020).

7. Bowen, K. J. \& Ebi, K. L. Governing the health risks of climate change: towards multi-sector responses. Curr. Opin. Environ. Sustain. 12, 80-85 (2015).

8. Olazabal, M., Ruiz de Gopegui, M., Tompkins, E. L., Vennerj, K. \& Smith, R. A cross-scale worldwide analysis of coastal adaptation planning. Environ. Res. Lett. 14, 124056 (2019).

9. England, M. I. et al. Climate change adaptation and cross-sectoral policy coherence in southern Africa. Reg. Environ. Change 18, 2059-2071 (2018).

10. Thomas, A., Shooya, O., Rokitzki, M., Bertrand, M. \& Lissner, T. Climate change adaptation planning in practice: insights from the Caribbean. Reg. Environ. Change 19, 2013-2025 (2019).

11. Canosa, I. V., Ford, J. D., McDowell, G., Jones, J. \& Pearce T, Progress in climate change adaptation in the Arctic. Environ. Res. Lett. 15, 2059-2071 (2020)

12. Robinson, $\mathrm{S}$. Adapting to climate change at the national level in Caribbean Small Island Developing States. Isl. Stud. J 13, 79-100 (2018).

13. Wirehn, L. Nordic agriculture under climate change: a systematic review of challenges, opportunities and adaptation strategies for crop production. Land Use Policy 77, 63-74 (2018).

14. Robinson, S. Climate change adaptation in SIDS: a systematic review of the literature pre and post the IPCC Fifth Assessment Report. WIREs Clim. Change 11, e653 (2020).

15. Lesnikowski, A. C. et al. National-level factors affecting planned, public adaptation to health impacts of climate change. Glob. Environ. Change 23, 1153-1163 (2013).

16. Lesnikowski, A., Ford, J. D., Biesbroek, R. \& Berrang-Ford, L. A policy mixes approach to conceptualizing and measuring climate change adaptation policy. Clim. Change 156, 447-469 (2019).

17. Hegger, D. L. T., Mees, H. L. P., Driessen, P. P. J. \& Runhaar, H. A. C. The roles of residents in climate adaptation: a systematic review in the case of the Netherlands. Environ. Policy Gov. 27, 336-350 (2017).

18. Lesnikowski, A. et al. Frontiers in data analytics for adaptation research: topic modeling. WIREs Clim. Change 10, e576 (2019).

19. Araos, M. et al. Climate change adaptation planning in large cities: a systematic global assessment. Environ. Sci. Policy 66, 375-382 (2016)

20. Singh, C., Madhavan, M., Arvind, J. \& Bazaz, A. Climate change adaptation in Indian cities: a review of existing actions and spaces for triple wins. Urban Clim. 36, 100783 (2021)

21. Lesnikowski, A., Biesbroek, R., Ford, J. D. \& Berrang-Ford, L. Policy implementation styles and local governments: the case of climate change adaptation. Environ. Polit. 30, 753-790 (2021).

22. Reckien, D. et al. Dedicated versus mainstreaming approaches in local climate plans in Europe. Renew. Sustain. Energy Rev. 112, 948-959 (2019).

23. Reckien, D. et al. How are cities planning to respond to climate change? Assessment of local climate plans from 885 cities in the EU-28. J. Clean. Prod. 191, 207-219 (2018).

24. Muchuru, S. \& Nhamo, G. Climate change and the African livestock sector. Emerging adaptation measures from UNFCCC national communications. Int. J. Clim. Change Strateg. Manag. 9, 241-260 (2017).

25. Lesnikowski, A. C., Ford, J. D., Berrang-Ford, L., Barrera, M. \& Heymann, J. How are we adapting to climate change? A global assessment. Mitig. Adapt. Strateg. Glob. Change 20, 277-293 (2015).

26. Gagnon-Lebrun, F. \& Agrawala, S. Implementing adaptation in developed countries: an analysis of progress and trends. Clim. Policy 7, 392-408 (2007). 
27. Regmi, B. R., Star, C. \& Leal Filho, W. An overview of the opportunities and challenges of promoting climate change adaptation at the local level: a case study from a community adaptation planning in Nepal. Clim. Change 138, 537-550 (2016).

28. Regmi, B. R., Star, C. \& Leal Filho, W. Effectiveness of the Local Adaptation Plan of Action to support climate change adaptation in Nepal. Mitig. Adapt. Strateg. Glob. Change 21, 461-478 (2016).

29. Leiter, T. in Adaptation Gap Report 2020 33-40 (UNEP, 2021).

30. Biesbroek, R. et al. Data, concepts and methods for large- $n$ comparative climate change adaptation policy research: a systematic literature review. WIREs Clim. Change 9, e548 (2018).

31. Biesbroek, R., Badloe, S. \& Athanasiadis, I. N. Machine learning for research on climate change adaptation policy integration: an exploratory UK case study. Reg. Environ. Change 20, 85 (2020).

32. Callaghan, M. W., Minx, J. C. \& Forster, P. M. A topography of climate change research. Nat. Clim. Change 10, 118-123 (2020)

33. Berrang-Ford, L., Pearce, T. \& Ford, J. D. Systematic review approaches for climate change adaptation research. Reg. Environ. Change 15, 755-769 (2015).

34. Minx, J. C., Callaghan, M., Lamb, W. F., Garard, J. \& Edenhofer, O. Learning about climate change solutions in the IPCC and beyond. Environ. Sci. Policy 77, 252-259 (2017).

35. Lamb, W. F., Callaghan, M. W., Creutzigt, F., Khosla, R. \& Minx, J. C. The literature landscape on $1.5^{\circ} \mathrm{C}$ climate change and cities. Curr. Opin. Environ. Sustain. 30, 26-34 (2018).

36. Lamb, W. F., Creutzig, F., Callaghan, M. W. \& Minx, J. C. Learning about urban climate solutions from case studies. Nat. Clim. Change $\mathbf{9}$, 279-287 (2019).

37. Sietsma, A. J., Ford, J. D., Callaghan, M. W. \& Minx, J. C. Progress in climate change adaptation research. Environ. Res. Lett. 16, 054038 (2021).

38. Ford, J. D. et al. Big data has big potential for applications to climate change adaptation. Proc. Natl Acad. Sci. USA 113, 10729-10732 (2016).

39. Cheng, S. H. et al. Using machine learning to advance synthesis and use of conservation and environmental evidence. Conserv. Biol. 32, 762-764 (2018).

40. Grubert, E. \& Siders, A. Benefits and applications of interdisciplinary digital tools for environmental meta-reviews and analyses. Environ. Res. Lett. 11, 093001 (2016).

41. Lukyanenko, R., Wiggins, A. \& Rosser, H. K. Citizen science: an information quality research frontier. Inf. Syst. Front. 22, 961-983 (2020).

42. Ziervogel, G., Pasquini, L. \& Lee, J. in University Initiatives in Climate Change Mitigation and Adaptation (eds Filho, W. L. \& Leal-Arcas, R.) 57-75 (Springer 2018); https://doi.org/10.1007/978-3-319-89590-14

43. Seltzer, E. \& Mahmoudi, D. Citizen participation, open innovation, and crowdsourcing: challenges and opportunities for planning. J. Plan. Lit. 28, 3-18 (2013)

44. Kueffer, C. et al. Enabling effective problem-oriented research for sustainable development. Ecol. Soc. 17, 8 (2012).

45. Haddaway, N. R. et al. On the use of computer-assistance to facilitate systematic mapping. Campbell Syst. Rev. 16, e1129 (2020).

46. Asfaw, A., Simane, B., Bantider, A. \& Hassen, A. Determinants in the adoption of climate change adaptation strategies: evidence from rainfed-dependent smallholder farmers in north-central Ethiopia (Woleka sub-basin). Environ. Dev. Sustain. 21, 2535-2565 (2019).

47. Bizikova, L., Parry, J.-E., Karami, J. \& Echeverria, D. Review of key initiatives and approaches to adaptation planning at the national level in semi-arid areas. Reg. Environ. Change 15, 837-850 (2015).

48. Oviedo, A. F. P., Mitraud, S., McGrath, D. G. \& Bursztyn, M. Implementing climate variability at the community level in the Amazon floodplain. Environ. Sci. Policy 63, 151-160 (2016).

49. Daryanto, S., Wang, L. \& Jacinthe, P.-A. Global synthesis of drought effects on cereal, legume, tuber and root crops production: a review. Agric. Water Manag. 179, 18-33 (2017).

50. Aggarwal, P., Vyas, S., Tornton, P. \& Campbell, B. M. How much does climate change add to the challenge of feeding the planet this century? Environ. Res. Lett 14, 043001 (2019).

51. Simpson, N. P., Shearing, C. D. \& Dupont, B. Climate gating: a case study of emerging responses to Anthropocene risks. Clim. Risk Manag. 26, 100196 (2019).

52. Simpson, N. P., Shearing, C. D. \& Dupont, B. Gated adaptation during the Cape Town drought: mentalities, transitions and pathways to partial nodes of water security. Soc. Nat. Resour. 33, 1041-1049 (2020).

53. Jacobi, J. et al. Actor-specific risk perceptions and strategies for resilience building in different food systems in Kenya and Bolivia. Reg. Environ. Change 19, 879-892 (2019).

54. Sain, G. et al. Costs and benefits of climate-smart agriculture: the case of the Dry Corridor in Guatemala. Agric. Syst. 151, 163-173 (2017).
55. Klock, C. \& Nunn, P. D. Adaptation to climate change in Small Island Developing States: a systematic literature review of academic research. J. Environ. Dev. 28, 196-218 (2019).

56. Mycoo, M. A. Beyond $1.5^{\circ} \mathrm{C}$ : vulnerabilities and adaptation strategies for Caribbean Small Island Developing States. Reg. Environ. Change 18, 2341-2353 (2018)

57. Nalau, J. et al. The role of Indigenous and traditional knowledge in ecosystem-based adaptation: a review of the literature and case studies from the Pacific Islands. Weather Clim. Soc. 10, 851-865 (2018).

58. Broto, V. C. \& Bulkeley, H. A survey of urban climate change experiments in 100 cities. Glob. Environ. Change 23, 92-102 (2013).

59. Beiler, M. O., Marroquin, L. \& McNeil, S. State-of-the-practice assessment of climate change adaptation practices across metropolitan planning organizations pre- and post-Hurricane Sandy. Transp. Res. A 88, 163-174 (2016).

60. Azevedo de Almeida, B. \& Mostafavi, A. Resilience of infrastructure systems to sea-level rise in coastal areas: impacts, adaptation measures, and implementation challenges. Sustainability 8, 1115 (2016).

61. Hintz, M. J., Luederitz, C., Lang, D. J. \& von Wehrden, H. Facing the heat: a systematic literature review exploring the transferability of solutions to cope with urban heat waves. Urban Clim. 24, 714-727 (2018).

62. Austin, S. E. et al. Public health adaptation to climate change in OECD countries. Int. J. Environ. Res. Public Health 13, 889 (2016).

63. Turek-Hankins, L. L. et al. Climate change adaptation to extreme heat: a global systematic review of implemented action. Oxford Open Clim. Change 1, kgab005 (2021)

64. Bauer, A. \& Steurer, R. Multi-level governance of climate change adaptation through regional partnerships in Canada and England. Geoforum 51, 121-129 (2014).

65. Becker, A., Ng, A. K. Y., McEvoy, D. \& Mullett, J. Implications of climate change for shipping: ports and supply chains. WIREs Clim. Change $\mathbf{9}$, e503 (2018).

66. Gautier, D., Denis, D. \& Locatelli, B. Impacts of drought and responses of rural populations in West Africa: a systematic review. WIREs Clim. Change 7, 666-681 (2016)

67. Koerth, J., Vafeidis, A. T. \& Hinkel, J. Household-level coastal adaptation and its drivers: a systematic case study review. Risk Anal. 37, 629-646 (2017).

68. Porter, J. J., Dessai, S. \& Tompkins, E. L. What do we know about UK household adaptation to climate change? A systematic review. Clim. Change 127, 371-379 (2014).

69. Ford, J. D., McDowell, G. \& Jones, J. The state of climate change adaptation in the Arctic. Environ. Res. Lett. 9, 104005 (2014).

70. Mayanja, M. N., Rubaire-Akiiki, C., Morton, J. \& Kabasa, J. D. Pastoral community coping and adaptation strategies to manage household food insecurity consequent to climatic hazards in the cattle corridor of Uganda. Clim. Dev. 12, 110-119 (2020).

71. Antwi-Agyei, P., Dougill, A. J. \& Stringer, L. C. Barriers to climate change adaptation: evidence from northeast Ghana in the context of a systematic literature review. Clim. Dev. 7, 297-309 (2015).

72. Lwasa, S. et al. Urban and peri-urban agriculture and forestry: transcending poverty alleviation to climate change mitigation and adaptation. Urban Clim. 7, 92-106 (2014)

73. Rasul, G., Pasakhala, B., Mishra, A. \& Pant, S. Adaptation to mountain cryosphere change: issues and challenges. Clim. Dev. 12, 297-309 (2020).

74. Robinson, S. Climate Change adaptation trends in Small Island Developing States. Mitig. Adapt. Strateg. Glob. Change 22, 669-691 (2017).

75. Goldstein, A., Turner, W. R., Gladstone, J. \& Hole, D. G. The private sector's climate change risk and adaptation blind spots. Nat. Clim. Change $\mathbf{9}$, 18-25 (2019).

76. Middelbeek, L., Kolle, K. \& Verrest, H. Built to last? Local climate change adaptation and governance in the Caribbean-the case of an informal urban settlement in Trinidad and Tobago. Urban Clim. 8, 138-154 (2014).

77. Koerth, J., Vafeidis, A. T., Carretero, S., Sterr, H. \& Hinkel, J. A. A typology of household-level adaptation to coastal flooding and its spatio-temporal patterns. SpringerPlus 3, 466 (2014).

78. Fischer, A. P. Adapting and coping with climate change in temperate forests. Glob. Environ. Change 54, 160-171 (2019).

79. Kattumuri, R., Ravindranath, D. \& Esteves, T. Local adaptation strategies in semi-arid regions: study of two villages in Karnataka. India Clim. Dev. 9, 36-49 (2017).

80. Arku, F. S. Local creativity for adapting to climate change among rural farmers in the semi-arid region of Ghana. Int. J. Clim. Change Strateg. Manag. 5, 418-430 (2013).

81. Wheeler, S. A. \& Marning, A. Turning water into wine: exploring water security perceptions and adaptation behaviour amongst conventional organic and biodynamic grape growers. Land Use Policy 82, 528-537 (2019). 
82. Fischer, A. P. Characterizing behavioral adaptation to climate change in temperate forests. Landsc. Urban Plan. 188, 72-79 (2019).

83. Dube, T., Mlilo, C., Moyo, P., Ncube, C. \& Phiri, K. Will adaptation carry the future? Questioning the long-term capacity of smallholder farmers' adaptation strategies against climate change in Gwanda District, Zimbabwe. J. Hum. Ecol. 61, 20-30 (2018).

84. Sereenonchai, S. \& Arunrat, N. Fisher's decisions to adopt adaptation strategies and expectations for their children to pursue the same profession in Chumphon Province, Thailand. Climate 7, 34 (2019).

85. Shaffril, H. A. M., Krauss, S. E. \& Samsuddin, S. F. A systematic review on Asian's farmers' adaptation practices towards climate change. Sci. Total Environ. 644, 683-695 (2018).

86. Asadu, A. N., Ozioko, R. I. \& Dimelu, M. U. information source and Indigenous adaptation strategies of cucumber farmers in Enugu State, Nigeria. J. Agric. Ext. 22, 136-146 (2018).

87. Schofield, D. \& Gubbels, F. Informing notions of climate change adaptation: a case study of everyday gendered realities of climate change adaptation in an informal settlement in Dar es Salaam. Environ. Urban. 31, 93-114 (2019).

88. Chengappa, P. G., Devika, C. M. \& Rudragouda, C. S. Climate variability and mitigation: perceptions and strategies adopted by traditional coffee growers in India. Clim. Dev. 9, 593-604 (2017).

89. Zinia, N. J. \& McShane, P. Ecosystem services management: an evaluation of green adaptations for urban development in Dhaka, Bangladesh. Landsc. Urban Plan. 173, 23-32 (2018).

90. Ekstrom, J. A., Bedsworth, L. \& Fencl, A. Gauging climate preparedness to inform adaptation needs: local level adaptation in drinking water quality in CA, USA. Clim. Change 140, 467-481 (2017).

91. Kirchhoff, C. J. \& Watson, P. L. Are wastewater systems adapting to climate change? J. Am. Water Resour. Assoc. 55, 869-880 (2019).

92. Johannsdottir, L., Davidsdottir, B., Goodsite, M. E. \& Olafsson, S. What is the potential and demonstrated role of non-life insurers in fulfilling climate commitments? A case study of Nordic insurers. Environ. Sci. Policy 38, 87-106 (2014).

93. Makate, C. \& Makate, M. Interceding role of institutional extension services on the livelihood impacts of drought tolerant maize technology adoption in Zimbabwe. Technol. Soc. 56, 126-133 (2019).

94. Valois, P., Caron, M., Gousse-Lessard, A.-S., Talbot, D. \& Renaud, J.-S. Development and validation of five behavioral indices of flood adaptation. BMC Public Health 19, 245 (2019).

95. Mubiru, D. N. et al. Climate trends, risks and coping strategies in smallholder farming systems in Uganda. Clim. Risk Manag. 22, 4-21 (2018)

96. Rankoana, S. A. Perceptions of climate change and the potential for adaptation in a rural community in Limpopo Province, South Africa. Sustainability 8, 672 (2016).

97. Fagariba, C. J., Song, S. \& Baoro, S. K. G. S. Climate Change adaptation strategies and constraints in Northern Ghana: evidence of farmers in Sissala West District. Sustainability 10, 1484 (2018).

98. Teklewold, H., Gebrehiwot, T. \& Bezabih, M. Climate smart agricultural practices and gender differentiated nutrition outcome: an empirical evidence from Ethiopia. World Dev. 122, 38-53 (2019).

99. Singh, C. et al. Interrogating 'effectiveness' in climate change adaptation: 11 guiding principles for adaptation research and practice. Clim. Dev. https:// doi.org/10.1080/17565529.2021.1964937 (2021).

100. Leiter, T. \& Pringle, P. in Adaptation Metrics: Perspectives on Measuring Aggregating and Comparing Adaptation Results (eds Christiansen, L. et al.) 29-48 (UNEP DTU Partnership, 2018).

101. Eriksen, S. et al. Adaptation interventions and their effect on vulnerability in developing countries: help, hindrance or irrelevance? World Dev. 141, 105383 (2021)

102. Tellman, B. et al. Adaptive pathways and coupled infrastructure: seven centuries of adaptation to water risk and the production of vulnerability in Mexico City. Ecol. Soc. 23, 105383 (2018).

103. Singh, C. Is participatory watershed development building local adaptive capacity? Findings from a case study in Rajasthan, India. Environ. Dev. 25, 43-58 (2018)

104. Maharjan, A. et al. Migration and household adaptation in climate-sensitive hotspots in South Asia. Curr. Clim. Change Rep. 6, 1-16 (2020).

105. McLeman, R. et al. Conceptual framing to link climate risk assessments and climate-migration scholarship. Clim. Change 165, 24 (2021).

106. Kaczan, D. J. \& Orgill-Meyer, J. The impact of climate change on migration: a synthesis of recent empirical insights. Clim. Change 158, 281-300 (2020).
107. Williams, P. et al. Feasibility assessment of climate change adaptation options across Africa: an evidence-based review. Environ. Res. Lett. 16, 073004 (2021).

108. Termeer, C. J. A. M., Dewulf, A. \& Biesbroek, G. R. Transformational change: governance interventions for climate change adaptation from a continuous change perspective. J. Environ. Plan. Manag. 60, 558-576 (2016).

109. Kates, R. W., Travis, W. R. \& Wilbanks, T. J. Transformational adaptation when incremental adaptations to climate change are insufficient. Proc. Natl Acad. Sci. USA 109, 7156-7161 (2012).

110. Wilson, R. S., Herziger, A., Hamilton, M. \& Brooks, J. S. From incremental to transformative adaptation in individual responses to climate-exacerbated hazards. Nat. Clim. Change 10, 200-208 (2020).

111. Gillard, R., Gouldson, A., Paavola, J. \& Van Alstine, J. Transformational responses to climate change: beyond a systems perspective of social change in mitigation and adaptation. WIREs Clim. Change 7, 251-265 (2016).

112. Pelling, M., O’Brien, K. \& Matyas, D. Adaptation and transformation. Clim. Change 133, 113-127 (2015).

113. Warner, K. et al. Characteristics of transformational adaptation in climate-land-society interactions. Sustainability 11, 356 (2019).

114. Few, R., Morchain, D., Spear, D., Mensah, A. \& Bendapudi, R Transformation, adaptation and development: relating concepts to practice. Palgrave Commun. 3, 17092 (2017).

115. Haapio, M. O., Wairiu, M., Gonzalez, R. \& Morrison, K. Transformation of rural communities: lessons from a local self-initiative for building resilience in the Solomon Islands. Local Environ. 23, 352-365 (2018).

116. Islam, M. M., Sallu, S., Hubacek, K. \& Paavola, J. Migrating to tackle climate variability and change? Insights from coastal fishing communities in Bangladesh. Clim. Change 124, 733-746 (2014).

117. Archer, D. Building urban climate resilience through community-driven approaches to development: experiences from Asia. Int. J. Clim. Change Strateg. Manag. 8, 654-669 (2016).

118. Hlahla, S. \& Hill, T. R. Responses to climate variability in urban poor communities in Pietermaritzburg, KwaZulu-Natal, South Africa. SAGE Open https://doi.org/10.1177/2158244018800914 (2018).

119. Puthucherril, T., Evans, S. \& Doelle, M. The role of the UNFCCC regime in ensuring effective adaptation in developing countries: lessons from Bangladesh. Int. J. Clim. Law 4, 327-352 (2014).

120. Morecroft, M. D. et al. Measuring the success of climate change adaptation and mitigation in terrestrial ecosystems. Science 366, eaaw9256 (2019).

121. Haunschild, R., Bornmann, L. \& Marx, W. Climate change research in view of bibliometrics. PLoS ONE 11, e0160393 (2016).

122. Nalau, J. \& Verrall, B. Mapping the evolution and current trends in climate change adaptation science. Clim. Risk Manag. 32, 100290 (2021).

123. Tonmoy, F. N., El-Zein, A. \& Hinkel, J. Assessment of vulnerability to climate change using indicators: a meta-analysis of the literature. WIREs Clim. Change 5, 775-792 (2014).

124. Restemeyer, B. \& Boogaard, F. C. Potentials and pitfalls of mapping nature-based solutions with the online citizen science platform climateScan Land 10, 5 (2021).

125. Disney, J., Bailey, D., Farrell, A. \& Taylor, A. Anecdata.org: An online citizen science platform for building climate resilient communities. IEEE Xplore https://doi.org/10.1109/OCEANS.2018.8604515 (2019).

126. Pecl, G. T. et al. Redmap Australia: challenges and successes with a large-scale citizen science-based approach to ecological monitoring and community engagement on climate change. Front. Mar. Sci. 6, 349 (2019).

127. Kythreotis, A. P. et al. Citizen social science for more integrative and effective climate action: a science-policy perspective. Front. Environ. Sci. 7, 10 (2019).

128. Owen, G. What makes climate change adaptation effective? A systematic review of the literature. Glob. Environ. Change 62, 102071 (2020).

129. van Valkengoed, A. M. \& Steg, L. Meta-analyses of factors motivating climate change adaptation behaviour. Nat. Clim. Change 9, 158-163 (2019).

130. Windfeld, E. J., Ford, J. D., Berrang-Ford, L. \& McDowell, G. How do community-level climate change vulnerability assessments treat future vulnerability and integrate diverse datasets? A review of the literature. Environ. Rev. 27, 427-434 (2019).

Publisher's note Springer Nature remains neutral with regard to jurisdictional claims in published maps and institutional affiliations.

(c) The Author(s), under exclusive licence to Springer Nature Limited 2021 
Lea Berrang-Ford $\mathbb{1}^{1 凶}$, A. R. Siders $\mathbb{C}^{2}$, Alexandra Lesnikowski ${ }^{3}$, Alexandra Paige Fischer ${ }^{4}$, Max W. Callaghan ${ }^{1,5}$, Neal R. Haddaway ${ }^{5,6,7}$, Katharine J. Mach ${ }^{8}{ }^{8,9}$, Malcolm Araos ${ }^{10}$, Mohammad Aminur Rahman Shah ${ }^{11}{ }^{11}$, Mia Wannewitz ${ }^{12}$, Deepal Doshi ${ }^{12}$, Timo Leiter ${ }^{13}$, Custodio Matavel (10 14, Justice Issah Musah-Surugu15,16, Gabrielle Wong-Parodi ${ }^{17}$, Philip Antwi-Agyei ${ }^{18}{ }^{18}$, Idowu Ajibade ${ }^{10}{ }^{19}$, Neha Chauhan ${ }^{\circledR 20}$, William Kakenmaster ${ }^{21}$, Caitlin Grady ${ }^{22}$, Vasiliki I. Chalastani ${ }^{23}$, Kripa Jagannathan ${ }^{4,24}$, Eranga K. Galappaththi ${ }^{25,26,27}$, Asha Sitati ${ }^{28}$, Giulia Scarpa ${ }^{29}$, Edmond Totin ${ }^{30}{ }^{30}$, Katy Davis', Nikita Charles Hamilton ${ }^{1}{ }^{31,32}$, Christine J. Kirchhoff ${ }^{33}$, Praveen Kumar ${ }^{34,35}$, Brian Pentz $\mathbb{1}^{36}$, Nicholas P. Simpson $\mathbb{1}^{37}$, Emily Theokritoff ${ }^{38,39}$, Delphine Deryng $\left(\mathbb{D}^{39}\right.$, Diana Reckien ${ }^{40}$, Carol Zavaleta-Cortijo ${ }^{41,42}$, Nicola Ulibarri ${ }^{43}$, Alcade C. Segnon ${ }^{44,45}$, Vhalinavho Khavhagali40, Yuanyuan Shang ${ }^{46,47,48}$, Luckson Zvobgo ${ }^{37}{ }^{37}$, Zinta Zommers ${ }^{49}$, Jiren Xu ${ }^{11}$, Portia Adade Williams ${ }^{50}$,

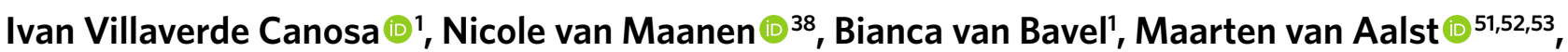
Lynée L. Turek-Hankins $\mathbb{1}^{54}$, Hasti Trivedi ${ }^{55}$, Christopher H. Trisos ${ }^{56}$, Adelle Thomas $\mathbb{1}^{38,57}$, Shinny Thakur ${ }^{58}$, Sienna Templeman ${ }^{59,60}$, Lindsay C. Stringer ${ }^{61}$, Garry Sotnik (iD ${ }^{4}$, Kathryn Dana Sjostrom ${ }^{62,63}$, Chandni Singh ${ }^{64}$, Mariella Z. Siña ${ }^{65}$, Roopam Shukla66, Jordi Sardans $\mathbb{1}^{67,68}$, Eunice A. Salubi ${ }^{69}$, Lolita Shaila Safaee Chalkasra ${ }^{70,71}$, Raquel Ruiz-Díaz ${ }^{72}$, Carys Richards ${ }^{73,74}$, Pratik Pokharel ${ }^{75,76}$, Jan Petzold ${ }^{12,77}$, Josep Penuelas ${ }^{67,68}$, Julia Pelaez Avila ${ }^{37}$, Julia B. Pazmino Murillo ${ }^{78}$, Souha Ouni ${ }^{79}$, Jennifer Niemann ${ }^{80}$, Miriam Nielsen ${ }^{85,60}$, Mark New ${ }^{37,81}$, Patricia Nayna Schwerdtle ${ }^{82,83,84}$, Gabriela Nagle Alverio ${ }^{85,86,87}$, Cristina A. Mullin (1D) ${ }^{88,89}$, Joshua Mullenite ${ }^{90}$, Anuszka Mosurska1, Mike D. Morecroft ${ }^{91}$, Jan C. Minx $\left({ }^{1,5}\right.$, Gina Maskell ${ }^{66}$, Abraham Marshall Nunbogu ${ }^{92}$, Alexandre K. Magnan ${ }^{103,94}$, Shuaib Lwasa ${ }^{95,96}$, Megan Lukas-Sithole ${ }^{56}$, Tabea Lissner ${ }^{38}$, Oliver Lilford ${ }^{97}$, Steven F. Koller ${ }^{54}$, Matthew Jurjonas ${ }^{98,99}$, Elphin Tom Joe ${ }^{100}$, Lam T. M. Huynh ${ }^{101}$, Avery Hill ${ }^{102}$, Rebecca R. Hernandez ${ }^{103,104}$, Greeshma Hegde ${ }^{105}$, Tom Hawxwell ${ }^{106,107}$, Sherilee Harper ${ }^{108}$, Alexandra Harden ${ }^{88}$, Marjolijn Haasnoot ${ }^{109,110}$, Elisabeth A. Gilmore ${ }^{111}$, Leah Gichuki ${ }^{112}$, Alyssa Gatt ${ }^{113}$, Matthias Garschagen ${ }^{114}$, James D. Ford ${ }^{1}{ }^{1}$, Andrew Forbes ${ }^{70}{ }^{70}$, Aidan D. Farrell ${ }^{115}$, Carolyn A. F. Enquist ${ }^{116}$, Susan Elliott ${ }^{92}$, Emily Duncan ${ }^{117}$, Erin Coughlan de Perez ${ }^{52,53,118}$, Shaugn Coggins ${ }^{119}$, Tara Chen ${ }^{120}$, Donovan Campbell' ${ }^{121}$, Katherine E. Browne ${ }^{6}{ }^{6}$, Kathryn J. Bowen ${ }^{122,123,124}$, Robbert Biesbroek ${ }^{125}$, Indra D. Bhatt ${ }^{58}$, Rachel Bezner Kerr ${ }^{126}$, Stephanie L. Barr ${ }^{609}$, Emily Baker ${ }^{126}$, Stephanie E. Austin ${ }^{127}$, Ingrid Arotoma-Rojas $\mathbb{1}^{1}$, Christa Anderson ${ }^{128}$, Warda Ajaz ${ }^{129}$, Tanvi Agrawal ${ }^{130}$ and Thelma Zulfawu Abu ${ }^{92}$

\footnotetext{
'Priestley International Centre for Climate, University of Leeds, Leeds, UK. ${ }^{2}$ Disaster Research Center, Biden School of Public Policy and Administration, Department of Geography and Spatial Sciences, University of Delaware, Newark, DE, USA. ${ }^{3}$ Department of Geography, Planning \& Environment, Concordia University, Montreal, Quebec, Canada. ${ }^{4}$ School for Environment and Sustainability, University of Michigan, Ann Arbor, MI, USA. ${ }^{5}$ Mercator Research Institute on Global Commons and Climate Change, Berlin, Germany. ${ }^{6}$ Stockholm Environment Institute, Stockholm, Sweden. ${ }^{7}$ Africa Centre for Evidence, University of Johannesburg, Johannesburg, South Africa. ${ }^{8}$ Department of Environmental Science and Policy, Rosenstiel School of Marine and Atmospheric Science, University of Miami, Miami, FL, USA. '̊eonard and Jayne Abess Center for Ecosystem Science and Policy, University of Miami, Coral Gables, FL, USA. ${ }^{10}$ Department of Sociology, New York University, New York, NY, USA. "'School of Interdisciplinary Studies, University of Glasgow, Dumfries, UK. ${ }^{12}$ Department of Geography, Ludwig-Maximilians-University Munich, Munich, Germany. ${ }^{13}$ Grantham Research Institute on Climate Change and the Environment, London School of Economics and Political Science, London, UK. ${ }^{14}$ Leibniz-Centre for Agricultural Landscape Research (ZALF), Müncheberg, Germany. ${ }^{15}$ United Nations University, Bonn, Germany. ${ }^{16}$ Department of Public Administration and Health Service Management, University of Ghana, Legon, Ghana. ${ }^{17}$ Department of Earth System Science and Woods Institute for the Environment, Stanford University, Stanford, CA, USA. ${ }^{18}$ Department of Environmental Science, Kwame Nkrumah University of Science and Technology, Kumasi, Ghana. ${ }^{19}$ Department of Geography, Portland State University, Portland, OR, USA. ${ }^{20}$ Faculty of Sustainability, Leuphana University, Lüneburg, Germany. ${ }^{21}$ University of Notre Dame, Dame, IN, USA. ${ }^{22}$ Department of Civil and Environmental Engineering, Rock Ethics Institute, Penn State University, University Park, PA, USA. ${ }^{23}$ Laboratory of Harbor Works, Department of Water Resources and Environmental Engineering, School of Civil Engineering, National Technical University of Athens, Zografou, Greece. ${ }^{24}$ Earth and Environmental Sciences Area, Lawrence Berkeley National Lab, Berkeley, CA, USA. ${ }^{25}$ Institute of Integrative Biology and Systems, Laval University, Quebec City, Quebec, Canada. ${ }^{26}$ Department of Geography, McGill University, Montreal, Quebec, Canada. ${ }^{27}$ Department of Geography, Virginia Tech, Blacksburg,
} 
VA, USA. ${ }^{28}$ United Nations Office for Disaster Risk Reduction, Geneva, Switzerland. ${ }^{29}$ School of Food and Nutrition, University of Leeds, Leeds, UK. ${ }^{30}$ École de Foresterie Tropicale, Université Nationale d'Agriculture, Kétou, Benin. ${ }^{31} \mathrm{NCH}$ Strategy Group, Nassau, Bahamas. ${ }^{32}$ The Department of Environmental Planning and Protection (DEPP), Nassau, Bahamas. ${ }^{33}$ Department of Civil \& Environmental Engineering, University of Connecticut, Storrs, CT, USA. ${ }^{34}$ School of Environmental Sciences, Jawaharlal Nehru University, New Delhi, India. ${ }^{35}$ Department of Sustainable Landscape Development, Martin Luther University Halle-Wittenberg, Halle (Saale), Germany. ${ }^{36}$ Department of Physical and Environmental Sciences, University of Toronto Scarborough, Toronto, Ontario, Canada. ${ }^{37}$ University of Cape Town, Cape Town, South Africa. ${ }^{38} \mathrm{Climate}$ Analytics, Berlin, Germany. ${ }^{39}$ Integrative Research Institute on Transformations of Human-Environment Systems (IRI THESys), Humboldt-Universität zu Berlin, Berlin, Germany. ${ }^{40}$ Faculty of Geo-Information Science and Earth Observation, Department of Urban and Regional Planning and Geo-Information Management, University of Twente, Enschede, the Netherlands. ${ }^{41}$ Facultad de Salud Pública y Administración, Universidad Peruana Cayetano Heredia, Lima, Perú. ${ }^{42}$ School of Food Science and Nutrition, Faculty of Environment, University of Leeds, Leeds, UK. ${ }^{43}$ Department of Urban Planning \& Public Policy, University of California Irvine, Irvine, CA, USA. ${ }^{44}$ Faculty of Agronomic Sciences, University of Abomey-Calavi, Cotonou, Benin. ${ }^{45}$ CGIAR Research Program on Climate Change, Agriculture and Food Security (CCAFS), International Crops Research Institute for the Semi-Arid Tropics (ICRISAT), Bamako, Mali. ${ }^{46}$ Australian National Centre for the Public Awareness of Science, The Australian National University, Canberra, Australian Capital Territory, Australia. ${ }^{47}$ Department of Government, University of Essex, Colchester, UK. ${ }^{48}$ Preqin Ltd, London, UK. ${ }^{49}$ United Nations Office for the Coordination of Humanitarian Affairs, New York, NY, USA. ${ }^{50}$ CSIR-Science and Technology Policy Research Institute, Accra, Ghana. ${ }^{51}$ Faculty of Geo-information Science and Earth Observation, University of Twente, Twente, the Netherlands. ${ }^{52}$ Red Cross Red Crescent Climate Centre, The Hague, The Netherlands. ${ }^{53}$ International Research Institute for Climate and Society, Columbia University, Palisades, NY, USA. ${ }^{4}$ Environmental Science and Policy Graduate Program, Leonard and Jayne Abess Center for Ecosystem Science and Policy, University of Miami, Coral Gables, FL, USA. ${ }^{55}$ Maharaja Sayajirao University of Baroda, Gujarat, India. ${ }^{56}$ African Climate and Development Initiative, University of Cape Town, Cape Town, South Africa. ${ }^{57}$ University of The Bahamas, Nassau, Bahamas. ${ }^{58} \mathrm{G}$. B. Pant National Institute of Himalayan Environment, Kosi-Katarmal, Almora, India. ${ }^{59}$ Department of Earth and Environmental Sciences, Columbia University, New York, NY, USA. ${ }^{60}$ National Aeronautics and Space Administration (NASA) Goddard Institute for Space Studies, New York, NY, USA. ${ }^{61}$ Department of Environment and Geography, University of York, York, UK. ${ }^{62}$ Memphis-Shelby County Division of Planning and Development, Memphis, TN, USA. ${ }^{63}$ University of Memphis, Memphis, TN, USA. ${ }^{64}$ School of Environment and Sustainability, Indian Institute for Human Settlements, Bangalore, India. ${ }^{65}$ School of Public Health and Administration, Cayetano Heredia University, Lima, Peru. ${ }^{66}$ Potsdam Institute for Climate Impact Research (PIK), Member of the Leibniz Association, Telegrafenberg, Potsdam, Germany. ${ }^{67} \mathrm{CSIC}$, Global Ecology Unit CREAF-CEAB-UAB, Bellaterra, Catalonia, Spain. ${ }^{68} \mathrm{CREAF}$, Cerdanyola del Vallès, Catalonia, Spain. ${ }^{6}$ University of Waterloo, Waterloo, Ontario, Canada. ${ }^{70}$ Department of Geography, Environment, and Geomatics, University of Ottawa, Ottawa, Ontario, Canada. ${ }^{71}$ International Development Research Centre, Ottawa, Ontario, Canada. ${ }^{72}$ Future Oceans Lab, CIM-Universidade de Vigo, Vigo, Spain. ${ }^{73}$ University of St Andrews, St Andrews, UK. ${ }^{74}$ University College London, London, UK. ${ }^{75}$ Danish Cancer Society Research Centre, Copenhagen, Denmark. ${ }^{76}$ Institute for Nutrition Research, School of Medical and Health Sciences, Edith Cowan University, Joondalup, Western Australia, Australia. ${ }^{77}$ Center for Earth System Research and Sustainability (CEN), University of Hamburg, Hamburg, Germany. ${ }^{78}$ Eberswalde University for Sustainable Development, Eberswalde, Germany. ${ }^{79}$ Columbia University, New York, NY, USA. ${ }^{80}$ Rosenstiel School of Marine and Atmospheric Science, University of Miami, Miami, FL, USA. ${ }^{81}$ University of East Anglia, Norwich, UK. ${ }^{82}$ Heidelberg Institute of Global Health (HIGH), Faculty of Medicine and University Hospital, Heidelberg University, Heidelberg, Germany. ${ }^{83}$ Nursing and Midwifery, Faculty of Medicine Nursing and Health Sciences, Monash University, Melbourne, Victoria, Australia. ${ }^{84}$ Medecins Sans Frontieres International, Geneva, Switzerland. ${ }^{85}$ Nicholas School of the Environment, Duke University, Durham, NC, USA. ${ }^{86}$ Sanford School of Public Policy, Duke University, Durham, NC, USA. ${ }^{87}$ School of Law, Duke University, Durham, NC, USA. ${ }^{88}$ Department of Civil \& Environmental Engineering and Department of Geography, University of Connecticut, Storrs, CT, USA. ${ }^{89}$ Oak Ridge Institute for Science and Education, Environmental Protection Agency, Washington, DC, USA. ${ }^{90}$ Wagner College, New York, NY, USA. ${ }^{91}$ Natural England, Worcester, UK. ${ }^{92}$ Department of Geography and Environmental Management, University of Waterloo, Waterloo, Ontario, Canada. ${ }^{93}$ Institute for Sustainable Development and International Relations, IDDRI-Sciences Po, Paris, France. ${ }^{94}$ LIENSs Laboratory, CNRS \& University of La Rochelle, La Rochelle, France. ${ }^{95} \mathrm{Global}$ Centre on Adaptation, Rotterdam, the Netherlands. ${ }^{96}$ Makerere University, Kampala, Uganda. ${ }^{97}$ Australian National University, Canberra, Australian Capital Territory, Australia. ${ }^{98}$ The Nature Conservancy, Michigan Chapter, Lansing, MI, USA. ${ }^{99}$ The Cooperative Institute for Great Lakes Research, Ann Arbor, MI, USA. ${ }^{100}$ Economics Center, World Resources Institute, New Delhi, India. ${ }^{101}$ Graduate Programme of Sustainability Science and Global Leadership Initiative, University of Tokyo, Tokyo, Japan. ${ }^{102}$ Department of Biology, Stanford University, Stanford, CA, USA. ${ }^{103}$ Department of Land, Air and Water Resources, University of California, Davis, CA, USA. ${ }^{104}$ The Wild Energy Initiative, Muir Institute of the Environment, University of California, Davis, CA, USA. ${ }^{105}$ Socratus Foundation for Collective Wisdom, Bangalore, India. ${ }^{106}$ Urban Planning and Regional Development, HafenCity University, Hamburg, Germany. ${ }^{107}$ Urban Systems Engineering, Fraunhofer Institute for Industrial Engineering (IAO), Stuttgart, Germany. ${ }^{108}$ University of Alberta, Edmonton, Alberta, Canada. ${ }^{109}$ Deltares, Delft, the Netherlands. ${ }^{110}$ Department of Physical Geography, Utrecht University, Utrecht, the Netherlands. "'Department of International Development, Community, and Environment, Clark University, Worcester, MA, USA. ${ }^{12}$ International Livestock Research Institute, Nairobi, Kenya. ${ }^{113}$ Wilfrid Laurier University, Waterloo, Ontario, Canada. ${ }^{114}$ Department of Geography, Ludwig-Maximilians University Munich, Munich, Germany. ${ }^{115}$ The University of the West Indies-St Augustine Campus, St George, Trinidad and Tobago. ${ }^{116}$ US Geological Survey, Southwest Climate Adaptation Science Center, Arizona, AZ, USA. ${ }^{17}$ Department of Geography, Environment, and Geomatics, University of Guelph, Guelph, Ontario, Canada. ${ }^{118}$ Friedman School of Nutrition Science and Policy, Tufts University, Boston, MA, USA. ${ }^{119}$ School of Public Health, University of Alberta, Edmonton, Alberta, Canada. ${ }^{120} \mathrm{E}-\mathrm{DA}$ Hospital, Kaohsiung, Taiwan. ${ }^{121}$ The University of West Indies, Mona, Jamaica. ${ }^{122}$ Fenner School of Environment and Society, Australian National University, Acton, Australian Capital Territory, Australia. ${ }^{123}$ Institute for Advanced Sustainability Studies, Potsdam, Germany. ${ }^{124}$ Melbourne School of Population and Global Health, University of Melbourne, Parkville, Victoria, Australia. ${ }^{125}$ Wageningen University, Wageningen, the Netherlands. ${ }^{126}$ Cornell University, Ithaca, NY, USA. ${ }^{127}$ Ostbayerische Technische Hochschule (OTH) Amberg-Weiden, Amberg, Germany. ${ }^{128}$ WWF, Washington DC, USA. ${ }^{129}$ USPCAS-E, National University of Sciences and Technology, Islamabad, Pakistan. ${ }^{130}$ Ashoka Trust for Research in Ecology and the Environment, Bangalore, India.

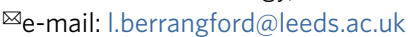




\section{Methods}

Methods protocols. Detailed protocols for this article are published via the Nature Protocol Exchange, and include Part 1-Introduction and overview of methods (https://doi.org/10.21203/rs.3.pex-1240/v1) 131, Part 2-Screening protocol (https:// doi.org/10.21203/rs.3.pex-1241/v1) 132 $^{132}$ and Part 3-Coding protocol (https://doi. org/10.21203/rs.3.pex-1242/v1 $)^{133}$. We additionally provide a backgrounder and reflective discussion of adaptation tracking and global mapping methodologies in Supplementary File 1. Detailed methods are provided that describe our assessment of the evidence of risk reduction (Supplementary File 2) and transformational adaptation (Supplementary File 3), which include confidence assessment and an internal expert elicitation exercise (Supplementary File 4). Full search strings are available in Supplementary File 5, and our full codebook is provided in Supplementary File 6.

Objectives and scope. We systematically assessed the global academic literature published between 2013 and 2019 to characterize human adaptation-related responses to climate change. We framed the review using standards to formulate research questions and searches in systematic reviews ${ }^{33,134}$, using a PICoST approach: population $(\mathrm{P})$, interest $(\mathrm{I})$, context $(\mathrm{Co})$, study design $(\mathrm{S})$ and time $(\mathrm{T})$.

The population $(\mathrm{P})$ includes all global human or natural systems of importance to humans that are impacted by climate change. The activity of interest (I) is adaptation-related responses. Owing to the lack of scientifically robust literature that assesses the potential effectiveness of responses, we use the term 'adaptation-related responses' rather than the more common 'adaptation' to avoid the implication that all the responses (or adaptations) are actually adaptive (that is, reduce vulnerability and/or risk); some responses labelled as 'adaptations' might, in fact, be maladaptive ${ }^{135}$. To be included, responses must be initiated by humans. This includes human-assisted responses within natural systems, as well as responses taken by governments, the private sector, civil society, communities, households and individuals, whether intentional and/or planned or unintentional and/or autonomous. Although unintentional and/or autonomous responses are included, these are likely to be under-represented unless the paper reporting them labelled them as adaptation or they were documented as a response to climate change. The document search for this review included search terms such as adaptation, resilience or risk management (Supplementary File 5), potentially not capturing activities not clearly identified as a response to climate changes. We excluded responses in natural systems that are not human assisted; these are sometimes referred to as evolutionary adaptations or autonomous natural systems adaptations ${ }^{136,137}$. Although important, autonomous adaptation in natural systems is distinct from adaptations initiated by humans; this review focuses on responses by humans to observed or projected climate change risk. We include any human responses to climate change impacts that are, or could, decrease vulnerability or exposure to climate-related hazards, as well as anticipatory measures in response to the expected impacts. We included papers in any language that were indexed (title, abstract and keywords) in English.

This review focuses on adaptation only, and excludes mitigation (responses that involve the reduction of greenhouse gas concentrations). We considered adaptation responses across contexts (Co) globally, and focused only on adaptation activities directly intended to reduce risk, exposure or vulnerability, even if later identified as maladaptation.

We focused on the academic literature only, which included empirical articles or reviews, data papers and letters, but excluded conference papers, book chapters and other non-journal document types. We excluded grey literature and other sources of Indigenous knowledge and local knowledge and practitioner knowledge. We focused on empirical literature only, which included qualitative or quantitative analysis and all study designs (S). To reflect publications since the Fifth Assessment Report and prior to the Sixth Assessment Report (AR6) publication cutoff, we focused on literature published in the time period (T) between 2013 and 2019.

This review responds to the mandate of the Intergovernmental Panel on Climate Change (IPCC) AR6 outline, which highlights the need to document and synthesize observed responses to climate change. Throughout this protocol, we drew on the foci, categorization and priorities outlined in the IPCC AR6 Working Group II outline (https://www.ipcc.ch/site/assets/uploads/2018/03/AR6_WGII_ outlines_P46.pdf) as a reflection of the stakeholder framing for this review. To maximize the potential impact of outputs, the timeline for this review was aligned with the publication schedule and publication cutoffs to inform the AR6 assessment process (https://www.ipcc.ch/report/sixth-assessment-report-working-group-ii/).

Summary of procedure. We followed guidelines for systematic evidence synthesis using the ROSES established reporting standards ${ }^{134}$. A summary of the documents screened and coded at different stages of the review is presented in Extended Data Fig. 1.

Database searches. Search strings were developed for each bibliographic database. The searches focused on documents that combined two concepts: climate change (climat ${ }^{*}$ or global warming) AND adaptation responses (adapt ${ }^{*}$ or resilien ${ }^{*}$ or risk management or risk reduction). Documents retrieved from the searches were uploaded to a customized platform for management and screening (https://doi.org/10.5281/zenodo.4121525). The search strings are detailed in Supplementary File 5.
Screening of documents. The objective of screening was to assemble a database of papers published between 2013 and 2019 on actions undertaken by people in response to climate change or environmental conditions, events and processes that were attributed or theorized to be linked, at least in part, to climate change. Inclusion criteria for screening are summarized in Extended Data Fig. 2.

The documents published between 2013 and 2019 included documents that reported on adaptations undertaken prior to 2013. Documents were not excluded from screening based on language as long as they were indexed in English. Documents were not excluded by geographical region, population, ecosystem, species or sector. Documents not indexed in Web of Science, Scopus or Medline as an article or review were not included. The focus was on adaptation; documents that focused on mitigation responses (that is, reducing greenhouse gas emissions) were excluded. Adaptation actions could take place at any level of social organization (individual, household, community, institution and government). Adaptation responses to perceived climate change impacts were eligible for inclusion. Documents that synthesized climate change impacts on populations but without an explicit and primary emphasis on adaptation responses were also excluded, except when climate responses were synonymous with climate impacts (for example, human migration or species shifts). Documents whose contributions were primarily conceptual or theoretical were treated as non-empirical and therefore excluded. We focused on documents that reported on responses that constituted adaptation based on a strict definition of the term: behaviours that directly aimed to reduce risk or vulnerability ${ }^{138}$. Documents that presented empirical syntheses of vulnerability or adaptive capacity without a primary or substantive focus on tangible adaptation responses (reactive or proactive) were excluded. Documents were considered eligible for inclusion if they explicitly documented adaptation actions that were theorized or conceptually linked to risk or vulnerability reduction. This excluded assessments of potential adaptation, intentions and/or plans to adapt and the discussion of adaptation constraints or barriers in the absence of documented actions that might reduce risk, exposure or vulnerability.

Supervised machine learning. We used supervised machine learning techniques to filter and prioritize the screening of documents that were most likely to meet the inclusion criteria ${ }^{31-33,35,36,39,139}$. This approach involved a small screening team ( $n=4$ people) manually screening (human coding) a subset of documents to 'teach' an automated classifier which documents are relevant according to a set of predefined criteria, and then this trained classifier used to predict the 'most likely to be relevant' literature.

Initial manual screening. We first screened a random sample of documents retrieved via the search strings. This sample of documents was reviewed by four screening team members; the documents that were labelled differently by different team members were then discussed until consensus was reached, to reduce bias and ensure consistency between team members. This initial phase created the first of several training samples used to train the machine learning algorithm to predict the relevant documents.

Iterative screening and training of the algorithm. This sample of manually screened documents was used to train a machine learning classifier to predict the relevance of the remaining documents. 'Predicted relevance' refers to the algorithmic likelihood that a particular article would be coded as 'relevant' based on the content of its title and abstract. Batches of documents with the highest predicted probability of relevance were then screened by hand, with iterative retraining of the classifier after each batch to continuously improve prediction. This meant that the screening team were able to prioritize the manual screening of articles most likely to be relevant to our inclusion criteria.

Assessment of 'borderline' documents. This iterative process continued until the classifier stopped predicting new relevant documents, and most documents being identified were then only borderline relevant. We thus did not manually screen every article, but did screen the majority of articles we predicted (via machine learning and the saturation of relevant articles during screening) to be relevant to our inclusion criteria.

Estimating the proportion of relevant documents retrieved through machine learning. We used a random sample of the remaining unscreened documents (which represent those that were rejected by our machine-learning-assisted process) to estimate how many of these documents might still be relevant, and completed the screening when the estimates indicated that the returns of additional screening would be low.

Performance statistics generated by the machine learning classifier showed negligible potential to increase recall further, which means that the remaining unscreened documents were likely to be: (1) not relevant and would be excluded if screened manually, (2) if relevant, would be borderline or marginally relevant or (3) relevant but include limited reference to key climate adaptation vocabulary (Extended Data Fig. 1). We can be confident that we retrieved at least $80 \%$ of the relevant articles; the $20 \%$ of articles that are not included are likely to comprise primarily articles that are borderline relevant. Our database thus includes a 
substantial portion of the scientific evidence base on observed adaptation responses globally.

Coding and data extraction. A total of 2,032 articles were retrieved from the screening stage and deemed potentially eligible for data extraction. The bibliographic information for articles that met the inclusion criteria during the screening were imported into the platform SysRev (https://sysrev.com). Given that the initial screening was conducted on titles and abstracts only, an additional screening step was undertaken during this phase (data extraction) to ensure the documents contained sufficient full-text information to extract relevant data. Thus, data extraction included two initial screening questions:

1. Is the document relevant according to inclusion or exclusion criteria? To verify the relevance of borderline inclusions.

2. Is there sufficient information detailed in the full text (a minimum of half a page of content that documents an adaptation-related response)? This question was used to screen out documents that referred to relevant adaptation responses in their title or abstract, but included no tangible detail or documentation within the article itself.

Bibliographic information for all the documents classified as relevant to inclusion criteria during screening were imported into SysRev. Extraction was undertaken by small teams of researchers based on regional and sectoral expertise. Each coder contributed to one or more teams based on their expertise. Recruitment of the coding team members aimed to ensure geographical and sectoral expertise aligned with relevant volumes of literature. Teams ranged, for example, from three coders with experience on adaptation in Small Island States to 30 coders with expertise in food-related adaptation. Literature on food security in Africa, for example, was reviewed by a team of researchers with expertise in African adaptation and/or food security. We developed an on-line training manual for coders. The manual included both contextual information on systematic review methodologies, as well as key details to guide data extraction, which included a detailed codebook. Non-English articles were coded by team members fluent in the language used (for example, French, Spanish, Portuguese and Mandarin). Our geographically diverse research team meant that we had sufficient language competency to assess all the articles that met the inclusion criteria.

Data extraction methods and codebook questions were developed in consultation with team members, informed by the literature on adaptation ${ }^{1,4,128}$, and guided by our key research questions: What climate hazards are driving responses? Who is responding? What types of responses are documented? Is adaptation reducing climate change risk? Are adaptations transformational?

Questions included both closed and restricted answer questions and open-ended narrative answer questions. The former facilitated quantitative categorical analysis (for example, descriptive statistics and summarizing studies in ordered tables) and the mapping of adaptation (breadth), whereas the latter facilitated a contextual understanding of adaptation and qualitative analysis. We classified responses based on global region and sector as per the IPCC AR6 outline. We categorized types of responses as behavioural and/or cultural, ecosystem based, institutional, and technological and/or infrastructural. We additionally considered evidence of transformational adaptation based on the dimensions of depth, scope, speed and challenges to adaptation limits. The coding of regional and sectoral foci within documents allowed a stratified analyses for individual sectors or regions. A copy of the full codebook, which includes all the variables and our operational definitions, is available in Supplementary File 6.

Quality assurance of coding. To enable cross-article comparisons, we conducted a quality assessment of each coder to identify those who had missed entries or skipped significant questions within the SysRev data extraction platform. Details of the quality assurance procedure are available at the Nature Protocol Exchange (https://doi.org/10.21203/rs.3.pex-1242/v1).

Reconciliation of double codes. To consolidate multiple responses into a single entry for each article, we used a script in R that followed a series of if/then statements (see protocol on the Nature Protocol Exchange, https://doi.org/10.21203/ rs.3.pex-1242/v1). A final database was compiled with a single line entry for each article. All the articles were assigned to IPCC regions based on the countries identified during coding. The final database contained 1,682 articles and 70 columns (70 data points for each article).

Synthesis. Geographical mapping. We used 'geoparsers' to classify documents based on their geographic focus. Geoparsers refer to algorithms that can extract geographical place names from text, based on dictionary methods or pretrained models. We employed geoparsers to determine the country of affiliation for the first author of the paper, as well as to identify which countries or places within countries are mentioned in the abstracts.

Descriptive summaries. We conducted basic descriptive statistics to estimate the total number of articles based on key restricted-answer variables, which included sector, region, hazard, actor and response type. We created simple bar charts and descriptive infographics.
Evidence of transformational adaptation. For each article included in this review, we coded the depth, scope, speed and challenge to limits of the adaptation response documented. We developed a table to define each element, and to define high, medium and low categories within each. We circulated this table to the GAMI advisory team and external reviewers to receive feedback and validate our definitions. A table that details the definitions of high, medium and low for each of the four elements is provided in Supplementary File 3. A small team of coders $(n=4)$ first coded 25 articles, reviewed their results, discussed discrepancies and refined the category definitions to ensure consistency. For each element (depth, scope, speed and limits), the coders also assessed the robustness of the evidence to support the designation as high, medium or low. This robustness score was based on: (1) whether the article addressed the particular element explicitly or whether information had to be inferred and (2) the quality of the evidence presented in the article (for example, sample size and confidence in methods). Papers could also be assessed as 'not applicable' or 'unable to assess' if the article provided insufficient information on the element in question (for example, speed). For each region* sector combination $(n=49)$, the team assessed the overall level (high, medium or low) for each component (depth, scope, speed and limits). These aggregation assessments were based on: (1) the number and percentage of papers that assessed each component for the sector ${ }^{\star}$ region combination, (2) relative agreement (variability) across papers within the sector ${ }^{*}$ region (for example, what percentage described a high depth adaptation) and (3) consideration of the robustness of the evidence for each component. The assessment of confidence in evidence was guided by the GRADE-Cerqual approach to evaluating confidence in qualitative evidence, adapted to the language of the IPCC's uncertainty guidance ${ }^{140,141}$.

If fewer than five studies were available for a particular assessment (for example, speed-Africa-health), either because there were too few papers in the region*sector, or because too few papers provided enough information to assess a given component, then the ranking in the final table was given as 'Insufficient information to assess'. If confidence in the evidence, based on agreement and robustness, was very low, no assessment was reported. Methods for confidence assessment are provided in Supplementary File 3, in particular in Table 3).

\section{Data availability}

Our a priori methodological protocol is registered (06-12-2019) and available via the OSF website ${ }^{142}$ : https://osf.io/ps6xj. We prepared a series of detailed methods to accompany this paper via the Nature Protocol Exchange, which include: Part 1-Introduction and overview of methods (https://doi.org/10.21203/rs.3.pex-1240/ v1) ${ }^{131}$, Part 2-Screening Protocol (https://doi.org/10.21203/rs.3.pex-1241/v1) ${ }^{132}$, and Part 3-Coding protocol (https://doi.org/10.21203/rs.3.pex-1242/v1) ${ }^{133}$. The data presented in this manuscript included survey extraction of information on adaptation from peer-reviewed articles.

\section{Code availability}

References to relevant coding are listed in Methods of this manuscript, and include a machine learning platform ${ }^{143}$ (https://doi.org/10.5281/zenodo.4121525) and reconciliation of $\operatorname{codes}^{144}$ (https://doi.org/10.5281/zenodo.4010763).

\section{References}

131. Berrang-Ford, L. et al. The Global Adaptation Mapping Initiative (GAMI): Part 1-Introduction and overview of methods. Protocol Exchange https:// doi.org/10.21203/rs.3.pex-1240/v1 (2021).

132. Fischer, A. P., et al. The Global Adaptation Mapping Initiative (GAMI): Part 2-Screening protocol. Protocol Exchange https://doi.org/10.21203/ rs.3.pex-1241/v1 (2021).

133. Lesnikowski, L. et al. The Global Adaptation Mapping Initiative (GAMI): Part 3-Coding protocol. Protocol Exchange https://doi.org/10.21203/ rs.3.pex-1242/v1 (2021).

134. Haddaway, N. R., Macura, B., Whaley, P. \& Pullin, A. S. ROSES RepOrting standards for Systematic Evidence Syntheses: pro forma, flow-diagram and descriptive summary of the plan and conduct of environmental systematic reviews and systematic maps. Environ. Evid. 7,7 (2018).

135. Magnan, A. K. et al. Addressing the risk of maladaptation to climate change. WIREs Clim. Change 7, 646-665 (2016).

136. Polechová, J. \& Barton, N. H. Limits to adaptation along environmental gradients. Proc. Natl Acad. Sci. USA 112, 6401-6406 (2015).

137. Boutin, S. \& Lane, J. E. Climate change and mammals: evolutionary versus plastic responses. Evol. Appl. 7, 29-41 (2014).

138. Lesnikowski, A. et al. Adapting to health impacts of climate change: a study of UNFCCC Annex I parties. Environ. Res. Lett. 6, 4 (2011).

139. Porciello, R., Ivanina, M., Islam, M., Einarson, S. \& Hirsch, H. Accelerating evidence-informed decision-making for the Sustainable Development Goals using machine learning. Nat. Mach. Intell. 2, 559-565 (2020).

140. Mastrandrea, M. D. et al. The IPCC AR5 guidance note on consistent treatment of uncertainties: a common approach across the working groups. Clim. Change 108, 675-691 (2011).

141. Lewis, S. et al. Applying GRADE-CERQual to qualitative evidence synthesis findings: introduction to the series. Implement. Sci. 13, 2 (2018). 
142. Berrang-Ford, L. et al. Global Adaptation Mapping Initiative (OSFHome, 2019); https://osf.io/ps6xj

143. Callaghan, M., Muller-Hansen, F., Hilaire, J., \& Lee, Y. T. NACOSOS: NLP assisted classification, synthesis and online screening (v.0.1.0). Zenodo https://doi.org/10.5281/zenodo.4121526 (2020).

144. Siders, A. R. GAMI intercoder reliability \& reconciliation. Zenodo https:// doi.org/10.5281/zenodo.4010763 (2020).

\section{Acknowledgements}

We thank the following individuals for contributions to various stages of this initiative: C. Abbey, C. Alarconr, S. Arowolo, K. Christopher, R. Cremades, E. Cremin, K. Dave, S. Davis, D. Die, S. D’haen, S. Gruza, T. Harrison, D. Heinrich, F. I. Hoefsloot, M. Hothman, K. Hou, J. Kumar, R. Lama, A. Mahanti, C. McOmber, A. Mukjerji, N. Nnebe, M. North, C. Ofeogbu, H. Panchal, S. Pandey, A. Pasha, J. Pathak, P. Shrestha, D. Singini, A. Srinidhi, C. Thangata, V. Thimmaiah, A. Welles, K. Wroten, A. Yue and K. Zhu. We thank T. Leuchtefeld and the SysRev team for extensive technical and design support in partnering with this initiative. This work was supported by the following funding grants: Agence Française de Développement (A.K.M.), UK Government Foreign, Commonwealth \& Development Office and the International Development Research Centre, Ottawa, Canada (109419-001, N.P.S.), Agence Nationale pour la Recherche (French National Research Agency, ANR-10-LABX-14-01, A.K.M.), Agence Nationale pour la Recherche (French National Research Agency, ANR-15-CE03-0003, A.K.M.), Studienstiftung des Deutsches Volkes (P.N.S.), UK Economic and Social Research Council (ESRC) Doctoral Training Partnership (ES/P000622/1, project reference 2098296, T.L.), National Science Foundation, Directorate for Geosciences (no. 1935961 E.A.G.), Social Science and Humanities Research Council (SSHRC) (756-2021-0037, E.K.G.), China Scholarship Council, Australian National University-Climate Change Institute Supplementary Scholarship (Y.S.), Ministerio de Ciencia, Innovación y Universidades (MICINN) (BIO-ARID PID2020-115770RB-I, J.S.), European Research Council (grant ERC-SyG-2013-610028, IMBALANCE-P, J. Petzold), Ministerio de Ciencia, Innovación y Universidades (MICINN) (ELEMENTALSHIFT PID2019110521GB-100, J. Penuelas), UGC-JRF scholarship, University Grants Commission, Ministry of Human Resource Development, Government of India (3643/(SC)(NET-DEC 2015, P.K.), National Institute for Health Research (NIHR) (using the UK's Official Development Assistance (ODA) Funding) and Wellcome under the NIHR-Wellcome Partnership for Global Health Research (218743/Z/19/Z, C.Z.-C), The Gulf Research Program of the National Academies of Sciences, Engineering, and Medicine (G.W.P.), International Development Research Centre Canada (L.S.S.C.), Agricultural Experiment Station Hatch projects (CA-R-A-6689-H and CA-D-LAW-2352-H, R.R.H.), German Ministry for Education and Research-ARIADNE project and IPCC-AR6-III-2 project (03SFK5J0 and 01LG1910A, J.M.), German Ministry for Education and ResearchARIADNE project (03SFK5J0, M.W.C.), NSF-CNH2-LRUI-ROA Grant, Equitable and Resilience Urban Socio-ecological Systems (no. 245531, I.A.-R.) and Portland State University Vision 2025 Grant (I.A.-R.).

\section{Author contributions}

The research was conceived, designed and led by a Coordination Team, which included L.B.-F., A.L., A.R.S., AP.F., M.W.C., N.R.H., J.C.M. and K.J.M. L.B.-F. led the overall project, M.W.C. led the machine learning, A.P.F. led the screening team, A.L. led the coding team, A.R.S. led the synthesis team and K.J.M. led the expert elicitation team. N.R.H. provided overall leadership and expertise in the systematic review methods. Expertise in machine learning and data science was provided by J.C.M. The following individuals comprised the Advisory Team, which reviewed, revised and refined the research design and protocols, aided the codebook development and conceptualization of the research, supported the team recruitment and guided alignment of the work with the IPCC timelines and methods: E. Totin, N.P.S., D. Deryng, D.R., M.v.A., C.H.T., A.T., L.C.S., C.S., M. New, M.D.M., J.C.M., A.K.M., S.L., T. Lissner, S.H., M.H., E.A.G., M.G., J.D.F., S.E., E.C.d.P., K.J.B., R.B. and R.B.K. The Screening Team screened all the documents for inclusion, and included I.V.C., G. Sotnik, M. Nielsen and A.P.F. The Coding Team conducted all the data extraction, and included: M.A., M.A.R.S., M.W., D. Doshi, T. Leiter, C.M., J.I.M.-S., G.W.-P., P.A.-A., I.A., N.C., W.K., C.G., V.I.C., K.J., E.K.G., A.S., G. Scarpa, E. Totin, K.D., N.C.H., C.J.K., P.K., B.P., N.P.S., E. Theokritof, D. Deryng, C.Z.-C., N.U., A.C.S., V.K., A.R.S., Y.S., L.Z., Z.Z., J.X., P.A.W., I.V.C., N.v.M., L.L.T.-H., H.T., S. Thakur, S. Templeman, K.D.S., M.Z.S., R.S., J.S., E.A.S., L.S.S.C., R.R.-D., C.R., P.P., J. Petzold, J. Penuelas, J.P.A., J.B.P.M., S.O., P.N.S., G.N.A., C.A.M., J Mullenite, A.M., G.M., A.M.N., M.L.-S., O.L., S.F.K., M.J., E.T.J., L.T.M.H., A. Harden, R.R.H., G.H., T.H., A. Hill, E.A.G., L.G., A.G., A.F., A.D.F., C.A.F.E., E.D., S.C., T.C., D.C., K.E.B., I.B., R.B.K., S.L.B., E.B., S.E.A., I.A.-R., C.A., W.A., T.A. and T.Z.A. The Synthesis Team conducted and led the synthesis of the results: A.R.S., T. Leiter, C.M., N.C., W.K., C.G., V.I.C., E.K.G., A.S., G. Scarpa, E. Totin, K.D., N.C.H., C.J.K., P.K., B.P., N.P.S., E. Theokritof, D. Deryng and D.R. The expert elicitation team comprised K.J., K.J.M., E. Totin and J.N. L.B.-F. led manuscript writing. All the team members contributed to manuscript development and revisions, and approved the final manuscript.

\section{Competing interests}

The authors declare no competing interests.

\section{Additional information}

Extended data is available for this paper at https://doi.org/10.1038/s41558-021-01170-y. Supplementary information The online version contains supplementary material available at https://doi.org/10.1038/s41558-021-01170-y.

Correspondence and requests for materials should be addressed to Lea Berrang-Ford. Peer review information Nature Climate Change thanks Minpeng Chen, Johanna Nalau, Emma Tompkins and the other, anonymous, reviewer(s) for their contribution to the peer review of this work.

Reprints and permissions information is available at www.nature.com/reprints. 
Human-screened $\mathrm{n}=4,500$

\section{Screened via}

machine learning $n=44,316$

2,032 human-screened AND predicted to be relevant by machine learning

\section{Human-coded (data extraction)} $n=5,383$ unique sets of code
Scopus
$n=36,183$
MEDLINE
$n=8,973$

$n=39,626$
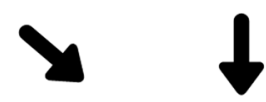

3

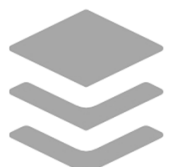

After duplicate removal

$$
n=48,816
$$

\section{Excluded}

$n=46,784$

Not climate change related Not adaptation related Not empirical Natural systems only Not response-oriented Historical focus Focus on planning only Predicted irrelevant

\section{Following title/abstract screening}

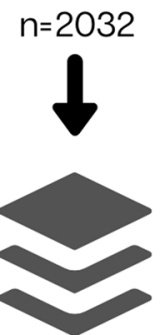

\section{Excluded} $n=350$

Insufficient data for analysis Not substantively empirical

Following full text screening

$$
n=1682
$$

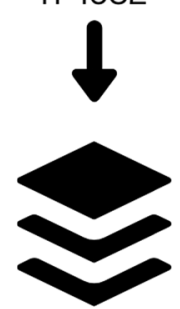

Coded in final database

$$
n=1682
$$

Extended Data Fig. 1 | Flowchart of GAMI database creation of papers published between 2013-2019 on actions undertaken by people in response to climate change or environmental conditions, events and processes that were attributed or theorized to be linked, at least in part, to climate change. 
Inclusion criteria

1 Climate change related

2 Adaptation

3 Empirical OR review of empirical

4 Human system responses OR human-assisted

5 Response-oriented, including factors in response

6 Recent or current (e.g. within past 10yrs)

7 Tangible responses with potential to directly reduce risk

\section{Exclusion criteria}

Not climate change related

Mitigation only

Conceptual, theoretical, simulated (without empirical component)

Sole focus on natural system responses not related to human adaptation

Vulnerability assessment OR impacts-focused only

Historic OR Prehistoric OR Projected

Planning, prioritizing, proposing responses, with no substantive focus on tangible responses OR Link to risk reduction tangential or unclear

Extended Data Fig. 2 | Summary of inclusion and exclusion criteria used for screening. 